\title{
ON REDUCTIVE AUTOMORPHISM GROUPS OF REGULAR EMBEDDINGS
}

\author{
GUIDO PEZZINI
}

\begin{abstract}
Let $G$ be a connected reductive complex algebraic group acting on a smooth complete complex algebraic variety $X$. We assume that $X$ is a regular embedding, a condition satisfied in particular by smooth toric varieties and flag varieties. For any set $\mathcal{D}$ of $G$-stable prime divisors, we study the action on $X$ of the $\operatorname{group} \operatorname{Aut}^{\circ}(X, \mathcal{D})$, the connected automorphism group of $X$ stabilizing all elements of $\mathcal{D}$. We determine a Levi subgroup $A(X, D)$ of $\operatorname{Aut}^{\circ}(X, \mathcal{D})$, and also relevant invariants of $X$ as a spherical $A(X, \mathcal{D})$-variety. As a byproduct, we obtain a complete description of the inclusion relation between closures of $A(X, D)$-orbits on $X$.
\end{abstract}

\section{INTRODUCTION}

In the 1970's Michel Demazure described the connected automorphism groups of two distinguished classes of algebraic varieties equipped with the action of a connected reductive group $G$ : complete homogeneous spaces (see [De77]), and smooth complete toric varieties (see [De70]).

These two classes of $G$-varieties admit a common generalization: the regular embeddings, here also called $G$-regular embeddings, defined independently in [BDP90 and Gi89]. With the additional assumption of completeness, Frédéric Bien and Michel Brion showed that these varieties form a relevant class of spherical varieties, which are by definition normal $G$-varieties with a dense orbit of a Borel subgroup of $G$.

The goal of this paper is to study the connected automorphism group $\operatorname{Aut}^{\circ}(X)$ of a complete regular embedding $X$. More precisely, we are interested in the group $\operatorname{Aut}^{\circ}(X, \mathcal{D})$, where $\mathcal{D}$ is any set of $G$-stable prime divisors of $X$, and $\operatorname{Aut}^{\circ}(X, \mathcal{D})$ is the connected automorphism group of $X$ stabilizing all elements of $\mathcal{D}$.

Our results are divided in several steps according to additional hypotheses on $\mathcal{D}$ and on $G$, and in each step we accomplish two goals. The first one is to describe a Levi subgroup $A(X, \mathcal{D})$ of $\operatorname{Aut}^{\circ}(X, \mathcal{D})$, based on the knowledge of discrete invariants associated with the $G$ action of $X$. These invariants come from the theory of spherical varieties, and are: the group $\Lambda_{G}(X)$ of $B$-eigenvalues of $B$-eigenvectors of $\mathbb{C}(X)$, the set of spherical roots $\Sigma_{G}(X)$ of $X$ (see Definition 2.4), the set $\Delta_{G}(X)$ of $B$-stable but not $G$-stable prime divisors of $X$, and the stabilizer $\mathrm{P}_{G}(X)$ of the open $B$-orbit of $X$. The last invariant is the fan $\mathcal{F}_{G}(X)$, a collection of strictly convex polyhedral cones in the vector space $\operatorname{Hom}_{\mathbb{Z}}\left(\Lambda_{G}(X), \mathbb{Q}\right)$ (see Definition 2.3).

2010 Mathematics Subject Classification. 14M27, 14M17, 14J50. 
The fan determines $X$ uniquely among the complete regular embeddings having the same open $G$-orbit, generalizing the fan associated with a toric variety. It also provides a combinatorial description of the $G$-orbits of $X$ and of the inclusion relation between $G$-orbit closures (see Kn91]).

Now $X$ is a spherical variety also under the action of $A(X, \mathcal{D})$, and our second goal is to determine the above invariants of $X$ with respect to the action of $A(X, \mathcal{D})$. In particular, this provides a combinatorial description of the $A(X, \mathcal{D})$-orbits on $X$.

Our approach is based on the analysis of a spherical variety $\mathbb{X}$ canonically associated with $X$ and equipped with a canonical $G$-equivariant map $X \rightarrow \mathbb{X}$. The variety $\mathbb{X}$, defined in Section 3 , is obtained from $X$ using a procedure called wonderful closure, which is closely related to the well-known construction of the spherical closure of a generic stabilizer of a spherical variety. We introduce the wonderful closure because it turns out to give much more direct informations on the automorphisms of $X$ than the spherical closure. On the other hand wonderful varieties such as $\mathbb{X}$ (see Definition 3.3) play a central role in the theory of spherical varieties (see e.g. [Lu01]), and their automorphism groups have been already studied in [Br07] and [Pe09].

Our study of the group $\operatorname{Aut}^{\circ}(X, \mathcal{D})$ proceeds by "successive approximation" with a sequence of subgroups that starts with elements very closely related to $\operatorname{Aut}^{\circ}(\mathbb{X})$. More precisely, we consider the filtration

$$
\operatorname{Aut}^{\circ}(X, \partial X) \subseteq \operatorname{Aut}^{\circ}\left(X, \mathcal{D} \cup(\partial X)^{\ell}\right) \subseteq \operatorname{Aut}^{\circ}(X, \mathcal{D})
$$

where we denote by $\partial X$ the set of all $G$-stable prime divisors of $X$ and by $(\partial X)^{\ell}$ the subset of $G$-invariant prime divisors mapping surjectively onto $\mathbb{X}$.

The group $\operatorname{Aut}^{\circ}(X, \partial X)$ is also the connected group of automorphisms stabilizing all $G$-orbits, and is the subject of Section 4. This group is reductive after [Br07] and $X$ is regular under its action; moreover $\operatorname{Aut}^{\circ}(X, \partial X)$ is equal to $\operatorname{Aut}^{\circ}(\mathbb{X}, \partial \mathbb{X})$ up to central isogeny and up to a torus factor. We recall that $\operatorname{Aut}^{\circ}(\mathbb{X}, \mathbb{D})$ for any $\mathbb{D} \subseteq \partial \mathbb{X}$ is reductive, and known after [Pe09].

After providing some technical results relating the automorphisms of $X$ and $\mathbb{X}$ in Sections 5 and 6, we devote $\operatorname{Section} 7$ to $\operatorname{Aut}^{\circ}\left(X, \mathcal{D} \cup(\partial X)^{\ell}\right)$. We show that this group is again reductive: it is equal to $\operatorname{Aut}^{\circ}(\mathbb{X}, \mathbb{D})$ up to central isogeny and up to a torus factor, where $\mathbb{D} \subseteq \partial \mathbb{X}$ is now the set of the images $\pi(D) \in \partial \mathbb{X}$ such that $D \in \partial X$ is stable under $\operatorname{Aut}^{\circ}\left(X, \mathcal{D} \cup(\partial X)^{\ell}\right)$.

This is the most technically involved part of the paper, and we use an indirect approach. First we analyze how the invariants of $X$ behave whenever $\operatorname{Aut}^{\circ}\left(X, \mathcal{D} \cup(\partial X)^{\ell}\right)$ is strictly bigger than $\operatorname{Aut}^{\circ}(X, \partial X)$. Under this hypothesis the fan $\mathcal{F}_{G}(X)$ has a particularly nice structure and is essentially determined by the subfan $\mathcal{F}_{G}^{\Lambda}(X) \subseteq \mathcal{F}_{G}(X)$ obtained intersecting all cones with a linear subspace naturally associated with $\mathbb{D}$ (see Lemma [7.2).

Then, using the open $\operatorname{Aut}^{\circ}(\mathbb{X}, \mathbb{D})$-orbit of $\mathbb{X}$ and the subfan $\mathcal{F}_{G}^{\Lambda}(X)$, we build ex novo a regular variety $X_{A}$ under the action of a reductive group $A$ which is equal up to a torus factor 
to the universal cover of $\operatorname{Aut}^{\circ}(\mathbb{X}, \mathbb{D})$ (see Proposition 7.13 ad Corollary 7.17). We show in Proposition 7.13 and Theorem 7.19 that $A$ is equal to $\operatorname{Aut}^{\circ}\left(X, \mathcal{D} \cup(\partial X)^{\ell}\right)$ up to central isogeny, and that $X_{A}$ is $A$-equivariantly isomorphic to $X$.

We study the group $\operatorname{Aut}^{\circ}(X, \mathcal{D})$ in Section 8 for any $\mathcal{D}$ assuming that $G$ is abelian, so $X$ is a smooth complete toric variety. Here $\mathbb{X}$ is least useful, because in this case it is a single point. However, the classical results by Demazure describe a Levi subgroup $A(X, \mathcal{D})$ of $\operatorname{Aut}^{\circ}(X, \mathcal{D})$, and it only remains to compute the invariants of $X$ as a spherical variety under its action (see Proposition 8.9 and Theorem 8.12). In particular $X$ is not necessarily regular under the action of $A(X, \mathcal{D})$, but we show that it is horospherical, i.e. it has no spherical root.

Section 9 deals with the case where $\mathcal{D}$ is again arbitrary but $G$ is semisimple. Our main result here is that $\operatorname{Aut}^{\circ}\left(X, \mathcal{D} \cup(\partial X)^{\ell}\right)$ is itself a Levi subgroup of $\operatorname{Aut}^{\circ}(X, \mathcal{D})$ (see Theorem 9.1). Therefore, in this case, our results of Section 7 accomplish our goals in full generality.

Finally, we discuss in Section 10 the group $\operatorname{Aut}^{\circ}(X, \mathcal{D})$ with no restriction on $\mathcal{D}$ or $G$. Here we build on our results on $\operatorname{Aut}^{\circ}\left(X, \mathcal{D} \cup(\partial X)^{\ell}\right)$ together with our results on the abelian case, which can be applied to a general fiber $Y$ of the map $\pi: X \rightarrow \mathbb{X}$ since $Y$ is a union of toric varieties.

We show in Propositions 10.1 and 10.4, that a Levi subgroup $A(X, \mathcal{D})$ is equal, up to isogeny, to the product of the commutator of $\operatorname{Aut}^{\circ}\left(X, \mathcal{D} \cup(\partial X)^{\ell}\right)$ with a reductive subgroup of $\operatorname{Aut}^{\circ}\left(X^{\prime}\right)$, where $X^{\prime}$ is a connected component of $Y$. We also compute the invariants of $X$ under the action of $A(X, \mathcal{D})$ in Proposition 10.7 and Theorem 10.10 .

Acknowledgements. I thank Jacopo Gandini for stimulating discussions, and I am especially grateful to Michel Brion for discussions and support during the development of this work. I thank the anonymous referees for useful remarks and suggestions on a previous version of this paper.

Notations. Through this paper $G$ is a connected reductive linear algebraic group over the field of complex numbers $\mathbb{C}$. We assume that $G=G^{\prime} \times C$ where $C$ is an algebraic torus and $G^{\prime}$ is semisimple and simply connected. We denote by $\mathbb{G}_{m}$ the one-dimensional torus.

We fix a Borel subgroup $B \subseteq G$ and a maximal torus $T \subseteq B$. We denote by $B^{-}$the Borel subgroup of $G$ such that $B \cap B^{-}=T$. If $\alpha$ is a root of $G$, then $\alpha^{\vee}$ denotes the corresponding coroot.

If $H$ is any algebraic group then we denote by $\mathrm{Z}(H)$ its center, by $H^{\circ}$ its connected component containing the unit element $e_{H}$, by $H^{r}$ its radical, by $H^{u}$ its unipotent radical, and by $\mathcal{X}(H)$ the set of its characters, i.e. algebraic group homomorphisms $H \rightarrow \mathbb{G}_{m}$. If $V$ is an $H$-module, then we denote by $V^{(H)}$ the set of non-zero $H$-semiinvariants of $V$, and for any $\chi \in \mathcal{X}(H)$ we 
set!

$$
V_{\chi}^{(H)}=\{v \in V \backslash\{0\} \mid h v=\chi(h) v \forall h \in H\} .
$$

If $H \subseteq K$ are subgroups of $G$, then we denote by $\pi^{H, K}: G / H \rightarrow G / K$ the natural map sending $g H \in G / H$ to $g K \in G / K$, and by $\mathrm{N}_{K} H$ the normalizer of $H$ in $K$.

For any subset $R$ of a $\mathbb{Z}$-module $\Lambda$, we denote by $R^{\geq 0}\left(\right.$ resp. $\left.R^{\perp}\right)$ the subset of $\operatorname{Hom}_{\mathbb{Z}}(\Lambda, \mathbb{Q})$ of all elements that are $\geq 0$ (resp. $=0$ ) on $R$. We define in the same way mutatis mutandis the subsets $R^{\geq 0}, R^{\perp} \subseteq \Lambda$ for $R \subseteq \operatorname{Hom}_{\mathbb{Z}}(\Lambda, \mathbb{Q})$. The notation $\langle-,-\rangle$ denotes the natural pairing between $\Lambda$ and $\operatorname{Hom}_{\mathbb{Z}}(\Lambda, \mathbb{Q})$.

If $X$ is an algebraic variety, the connected component containing $\operatorname{id}_{X}$ of its automorphism group is denoted by $\operatorname{Aut}^{\circ}(X)$. If a connected algebraic group $H$ acts on $X$, we denote by

$$
\theta_{H, X}: H \rightarrow \operatorname{Aut}^{\circ}(X)
$$

the corresponding homomorphism.

If $X$ is a $G$-variety, we denote by $\operatorname{Pic}^{G}(X)$ the group of isomorphism classes of $G$-linearized invertible sheaves. If $X$ is normal and $Y$ is a Cartier divisor, then the invertible sheaf $\mathcal{O}_{X}(Y)$ admits a (non unique) G-linearization (see [KKLV89, Remark after Proposition 2.4]). Through the paper $X$ will be in addition smooth, complete and quasi-homogeneous, and we will consider $G$-stable prime divisors. Then we may and will always assume that the $G$-linearization is chosen in such a way that the induced $G$-action on $H^{0}\left(X, \mathcal{O}_{X}(Y)\right)$ is equal to the action induced by the inclusion $H^{0}\left(X, \mathcal{O}_{X}(Y)\right) \subseteq \mathbb{C}(X)$.

\section{Complete Regular embeddings}

Definition 2.1. Let $X$ be an irreducible $G$-variety with an open $G$-orbit. Then $X$ is a $G$-regular embedding if for any $x \in X$ :

(1) the closure $\overline{G x}$ of its orbit is smooth, and if it is of positive codimension then it is the transversal intersection of the $G$-stable prime divisors containing it;

(2) the stabilizer $G_{x}$ has a dense orbit on the normal space in $X$ to the orbit $G x$ in the point $x$.

A $G$-regular embedding is smooth and has a finite number of $G$-orbits. Examples of $G$ regular embeddings are the $G$-homogeneous spaces for any $G$, and if $G$ is an algebraic torus then any smooth toric $G$-variety. Regular varieties occur naturally also in the theory of spherical varieties, i.e. normal $G$-varieties with a dense $B$-orbit.

\footnotetext{
${ }^{1}$ We underline that in our notation $V^{(H)}$ does not contain 0 and the same holds for $V_{\chi}^{(B)}$, in contrast with the similar common notation $V_{\chi}$ which we don't use here.
} 
More precisely, suppose that a $G$-variety $X$ is smooth and complete. Then $X$ is $G$-regular if and only if it is spherical and toroidal, i.e. any $B$-stable prime divisor containing a $G$-orbit is also $G$-stable (see [BB96, Proposition 2.2.1]).

We review some relevant invariants associated to any spherical $G$-variety $X$. If $x_{0}$ is a point on the open $G$-orbit of $X$, then we also denote the orbit $G x_{0}$ simply by $G / H$, where $H=G_{x_{0}}$ is called a generic stabilizer of $X$. In this case, $H$ is also called a spherical subgroup, and $\left(X, x_{0}\right)$ (or simply $X$ ) is called an embedding of $G / H$. A morphism between two embeddings $\left(X, x_{0}\right)$ and $\left(X^{\prime}, x_{0}^{\prime}\right)$ is a $G$-equivariant map $X \rightarrow X^{\prime}$ sending $x_{0}$ to $x_{0}^{\prime}$.

We will always assume that $x_{0}$ is chosen in such a way that $B x_{0}$ is dense in $X$. Then $H$ is also called a $B$-spherical subgroup.

Definition 2.2. Let $X$ be a spherical $G$-variety with open $G$-orbit $G / H$.

(1) We definet the lattice

$$
\Lambda_{G}(X)=\left\{\chi \in \mathcal{X}(B) \mid \mathbb{C}(X)_{\chi}^{(B)} \neq \varnothing\right\},
$$

whose rank is by definition the rank of $X$.

(2) We define

$$
\mathrm{N}_{G}(X)=\operatorname{Hom}_{\mathbb{Z}}\left(\Lambda_{G}(X), \mathbb{Q}\right) .
$$

(3) We define $\Delta_{G}(X)$ to be the set of colors of $X$, i.e. the $B$-stable prime divisors of $X$ having non-empty intersection with the open $G$-orbit of $X$.

(4) For any discrete valuation $\nu: \mathbb{C}(X) \backslash\{0\} \rightarrow \mathbb{Q}$ we define an element $\rho_{G, X}(\nu) \in \mathrm{N}_{G}(X)$ with the formula

$$
\left\langle\rho_{G, X}(\nu), \chi\right\rangle=\nu\left(f_{\chi}\right)
$$

where $f_{\chi} \in \mathbb{C}(X)_{\chi}^{(B)}$. If $D$ is a prime divisor of $X$ and $\nu_{D}$ is the associated discrete valuation, then we will also write $\rho_{G, X}(D)$ for $\rho_{G, X}\left(\nu_{D}\right)$.

(5) We define

$$
\mathrm{V}_{G}(X)=\left\{\rho_{G, X}(\nu) \mid \nu \text { is } G \text {-invariant }\right\},
$$

which is a polyhedral convex cone of maximal dimension in $\mathrm{N}_{G}(X)$ (see [Br90]); we denote its linear part by $\mathrm{V}_{G}^{\ell}(X)$.

(6) We define the boundary of $X$, denoted by $\partial_{G} X$, to be the set of the irreducible components of $X \backslash(G / H)$.

(7) We define $\mathrm{P}_{G}(X)$ to be the stabilizer of the open $B$-orbit of $X$. The set of simple roots associated to $\mathrm{P}_{G}(X)$ is denoted by $\mathrm{S}_{G}^{p}(X)$.

\footnotetext{
${ }^{2}$ We ignore the dependence on $B$ of all the invariants we define. This is justified by the fact that for any reductive group under consideration the choice of a Borel subgroup will be either unique (when the group is abelian) or always explicitly fixed.
} 
For the above, and for all the invariants defined later, we will drop the indices $G$ or $X$ whenever it is clear which group or which variety are considered. In loose terms the colors of $X$ can also be considered as invariants under $G$-equivariant birational maps, since they are the closures in $X$ of the colors of $G / H$.

The Luna-Vust theory of embeddings of homogeneous spaces specializes for spherical toroidal varieties in the following way (for details and proofs see [Kn96]).

Definition 2.3. Let $X$ be a toroidal spherical $G$-variety, and $Y$ an irreducible $G$-stable locally closed subvariety. Then we define $c_{X, Y} \subseteq \mathrm{N}(X)$ to be the polyhedral convex cone generated by $\rho\left(D_{1}\right), \ldots, \rho\left(D_{n}\right)$, where $D_{1}, \ldots, D_{n}$ are the $B$-stable prime divisors containing $Y$. The fan of $X$ is defined as

$$
\mathcal{F}_{G}(X)=\left\{c_{X, Y} \mid Y \text { a } G \text {-orbit of } X\right\}
$$

Notice that since $X$ is toroidal then the divisors $D_{1}, \ldots, D_{n}$ above are also $G$-stable for any $Y$. The collection of convex cones $\mathcal{F}(X)$ satisfies the following properties:

(1) each cone of $\mathcal{F}(X)$ is contained in $\mathrm{V}(G / H)$, it is strictly convex, and all its faces belong to $\mathcal{F}(X)$,

(2) any element of $\mathrm{V}(G / H)$ belongs to the relative interior of at most one cone of $\mathcal{F}(X)$.

The map $X \mapsto \mathcal{F}(X)$ induces a bijection between toroidal embeddings of $G / H$ (up to isomorphism of embeddings) and fans, i.e. collections of strictly convex polyhedral convex cones satisfying (1) and (2).

The support of a fan $\mathcal{F}$ is defined as

$$
\operatorname{supp} \mathcal{F}=\bigcup_{c \in \mathcal{F}} c
$$

The variety $X$ is complete if and only if $\operatorname{supp} \mathcal{F}(X)=\mathrm{V}(X)$, and it is smooth if and only if for each $c \in \mathcal{F}(X)$ there exists a basis $\gamma_{1} \ldots, \gamma_{r}$ of $\Lambda(X)$ and an integer $k$ between 1 and $r$ such that

$$
c=\left\{\gamma_{1}, \ldots, \gamma_{k}\right\}^{\geq 0}
$$

For later reference, we recall that if a spherical embedding $X$ is not toroidal, then it is also described by a similar datum, called a fan of colored convex cones. Here, the convex cone associated to a $G$-orbit $Y \subseteq X$ is replaced by the pair $\left(c_{X, Y}, d_{X, Y}\right)$ where $d_{X, Y}$ is the set of colors containing $Y$, and $c_{X, Y}$ is defined as above.

In general, the set $\mathrm{V}(X)$ is also a polyhedral convex cone of maximal dimension in $\mathrm{N}_{G}(X)$, and its linear part $\mathrm{V}^{\ell}(X)$ has dimension (as a $\mathbb{Q}$-vector space) equal to the dimension of $\mathrm{N}_{G} H / H$ (as a complex algebraic group). The equations defining the maximal proper faces of 
$\mathrm{V}(X)$ are linearly independent (see [Br90, Corollaire 3.3]). In other words, there always exist $\sigma_{1}, \ldots, \sigma_{k} \in \Lambda(X)$ that are indivisible, linearly independent, and such that

$$
\mathrm{V}(X)=\left\{-\sigma_{1}, \ldots,-\sigma_{k}\right\}^{\geq 0} .
$$

Definition 2.4. The elements $\sigma_{1}, \ldots, \sigma_{k}$ above are uniquely determined by $G / H$ and called the spherical roots of $X$; their set is denoted by $\Sigma_{G}(X)$.

The map $Y \mapsto c_{X, Y}$ sends a $G$-orbit of codimension $d$ in $X$ to a cone of dimension $d$, and this restricts to a bijection between the boundary $\partial X$ and the set of 1-dimensional cones in $\mathcal{F}(X)$.

Whether the cone of a given prime divisor $D \in \partial X$ lies or not on the linear part of the valuation cone has a strong influence on the automorphisms of $X$ not stabilizing $D$. This motivates the following definition.

Definition 2.5. For a subset $\mathcal{D} \subseteq \partial X$, we define the subsets

$$
\mathcal{D}^{\ell}=\left\{Y \in \mathcal{D} \mid c_{X, Y} \subset \mathrm{V}^{\ell}(X)\right\}
$$

and

$$
\mathcal{D}^{n \ell}=\mathcal{D} \backslash \mathcal{D}^{\ell}
$$

Example 2.6. Consider $G=\mathrm{SL}(n+1)$ (with $n \geq 2$ ) acting linearly and diagonally on $\mathbb{P}^{n+1} \times$ $\left(\mathbb{P}^{n}\right)^{*}$, where on the first factor it acts only on the first $n+1$ homogeneous coordinates. Choose $B$ to be the subgroup of upper triangular matrices. Then $X=\mathrm{Bl}_{p}\left(\mathbb{P}^{n+1}\right) \times\left(\mathbb{P}^{n}\right)^{*}$, with $p=$ $[0, \ldots, 0,1]$, is a $G$-regular variety with three $G$-stable prime divisors. It has rank 2 , and $\Lambda_{G}(X)$ is generated by the first and the last fundamental dominant weights, denoted resp. $\omega_{1}^{G}$ and $\omega_{n}^{G}$. The variety $X$ has two colors, namely the inverse images of the $B$-stable hyperplanes of its two factors. With respect to the basis of $\mathrm{N}_{G}(X) \cong \mathbb{Q}^{2}$ dual to $\left(\omega_{1}^{G}, \omega_{n}^{G}\right)$, the two colors have valuations $(1,0)$ and $(0,1)$, and the $G$-stable prime divisors $D_{1}, D_{2}$ and $E$ have valuations resp. $(-1,1),(1,-1)$ and $(-1,0)$. The fan $\mathcal{F}_{G}(X)$ has two maximal cones, generated resp. by $\rho\left(D_{1}\right)$, $\rho(E)$ and by $\rho(E), \rho\left(D_{2}\right)$, and $X$ has exactly one spherical root, namely $\sigma=\omega_{1}+\omega_{n}$. The divisor $E$ is the unique $G$-stable prime divisor not lying on the linear part of the valuation cone.

\section{Spherical AND WONDERful ClOSURE}

In this section we recall the notion, introduced in [Lu01], of the spherical closure $\bar{H}$ of a spherical subgroup $H \subseteq G$. We also define another subgroup containing $H$, called its wonderful closure. This is essentially already known, but not yet found in the literature.

Since we only consider the group $G$, in this section we drop all subscripts of the invariants of Definition 2.2, 
An element $n$ of the normalizer $\mathrm{N}_{G} H$ of $H$ induces a $G$-equivariant isomorphism $G / H \rightarrow G / H$ given by $g H \mapsto g n H$. This induces an action of $\mathrm{N}_{G} H$ on the set of colors $\Delta(G / H)$ : the spherical closure $\bar{H}$ of $H$ is defined as the kernel of this action.

If $\bar{H}=H$ then $H$ is called spherically closed, and for any spherical subgroup $H \subseteq G$ the spherical closure $\bar{H}$ is itself spherically closed. This is well known, and also follows easily from [BL11, Lemma 2.4.2]; we provide here a direct proof.

Proposition 3.1. For any spherical subgroup $H \subseteq G$, the spherical closure $\bar{H}$ is spherically closed.

Proof. Since $\bar{H}$ is contained in $\mathrm{N}_{G} H$ the quotient $\bar{H} / H$ is diagonalizable (see [Kn94, Theorem 6.1]), and thus $H$ is defined inside $\bar{H}$ as intersection of kernels of some characters. The colors of $G / \bar{H}$ generate $\operatorname{Pic}^{G}(G / \bar{H})$ (see [Br89, Proposition 2.2]) and the latter is isomorphic to $\mathcal{X}(\bar{H})$ (see [KKV89, Section 3.1]), therefore $\overline{\bar{H}}$ acts trivially on $\mathcal{X}(\bar{H})$.

This implies that $\overline{\bar{H}}$ normalizes $H$. By definition, it fixes all colors of $G / \bar{H}$, but these correspond to the colors of $G / H$ via the natural map $\pi^{H, \bar{H}}: G / H \rightarrow G / \bar{H}$. Hence $\overline{\bar{H}} \subseteq \bar{H}$.

For later convenience we report the following auxiliary result. Recall that whenever $H \subseteq K$ are spherical subgroups of $G$, the lattice $\Lambda(G / K)$ is contained in the lattice $\Lambda(G / H)$, since $B$ semiinvariant functions can be lifted from $G / K$ to $G / H$ via the map $\pi^{H, K}: G / H \rightarrow G / K$. We also denote this inclusion as a map $\left(\pi^{H, K}\right)^{*}: \Lambda(G / K) \rightarrow \Lambda(G / H)$, which induces a surjection $\pi_{*}^{H, K}: \mathrm{N}(G / H) \rightarrow \mathrm{N}(G / K)$.

Lemma 3.2. Let $H \subseteq K \subseteq \bar{H}$ be spherical subgroups of $G$. Then

$$
\left(\pi_{*}^{H, K}\right)^{-1}(\mathrm{~V}(G / H))=\mathrm{V}(G / K) .
$$

Proof. The claim stems from $\pi_{*}^{H, K}(\mathrm{~V}(G / H))=\mathrm{V}(G / K)$ together with $\operatorname{ker} \pi_{*}^{H, \bar{H}}=\mathrm{V}^{\ell}(G / H)$ (see [Kn96, Theorem 4.4 and Theorem 6.1]) and

$$
\operatorname{ker}\left(\pi_{*}^{H, K}\right) \subseteq \mathrm{V}^{\ell}(G / H)
$$

which follows from $\pi_{*}^{K, \bar{H}} \circ \pi_{*}^{H, K}=\pi_{*}^{H, \bar{H}}$.

A class of subgroups slightly broader then the spherically closed ones is the following.

Definition 3.3. If $\Sigma(G / H)$ is a basis of $\Lambda(G / H)$ then $H$ is called a wonderful subgroup of $G$. In this case there exists a fan having only one maximal cone equal to $\mathrm{V}(G / K)$; the associated toroidal embedding is denoted by $\mathbb{X}(G / H)$.

If $H$ is wonderful then the embedding $\mathbb{X}(G / H)$ is smooth, has a unique closed $G$-orbit and it is a wonderful variety in the sense of [Lu01]. A fundamental theorem of Knop (see [Kn96. Corollary 7.6]) states that a spherically closed subgroup is wonderful. 
Example 3.4. The converse of the above statement is false: for example, if $G=\operatorname{SO}(2 n+1)$ with $n \geq 2$, then $H=\mathrm{SO}(2 n)$ is a wonderful subgroup, with $\bar{H}=\mathrm{N}_{\mathrm{SO}(2 n+1)} \mathrm{SO}(2 n) \neq H$ (see [Wa96, Cases 7B, 8B of Table 1]).

It is possible to define canonically a minimal wonderful subgroup $\widehat{H}$ between $H$ and $\bar{H}$. As a byproduct, the automorphism groups of regular embeddings of $G / H$ are more directly related to the automorphism group of $\mathbb{X}(G / \widehat{H})$ than to that of $\mathbb{X}(G / \bar{H})$.

Definition 3.5. Let $H$ and $I$ be spherical subgroups of $G$. Then $I$ is a wonderful closure of $H$ if it is wonderful, satisfies $H \subseteq I \subseteq \bar{H}$, and is minimal with respect to these properties.

Example 3.6. Any wonderful subgroup $H \subseteq G$ is its own wonderful closure. We give an example of $H$ having wonderful closure different from $H$ and from $\bar{H}$. Let $G$ be $\operatorname{SO}(9)$, and let $P$ be a parabolic subgroup of $G$ with Levi subgroup $L=\operatorname{GL}(2) \times \operatorname{SO}(5)$. Set $L_{H}=$ $\mathrm{SL}(2) \times \mathrm{SO}(4) \subset L$, and let $H^{u} \subset P^{u}$ be the subgroup stable under conjugation by $L_{H}$ and such that $P^{u} / H^{u}$ is equivariantly isomorphic to $\mathbb{C}^{2} \otimes \mathbb{C}$ under the action of $L_{H}$. Then $H=L_{H} H^{u}$ is a spherical subgroup of $G$; it is not wonderful because it has infinite index in its normalizer. Consider now the subgroup $L_{K}=\mathrm{GL}(2) \times \mathrm{SO}(4)$, and $K=L_{K} H^{u}$. The group $K$ is minimal among the subgroups of $G$ strictly containing $H$ and of finite index in their normalizers. In addition $K$ is wonderful of rank 2 (see [Wa96, First case 6 of Table 2]), so it is a wonderful closure of $H$. On the other hand the normalizer of $H$ is equal to $\bar{H}$ (see [Wa96, Second case 6 of Table 2]), and $\bar{H}$ contains $K$ strictly (indeed $\left[N_{G} H: K\right]=2$ ).

We will show that a wonderful closure always exists and is unique; for this we need to recall a combinatorial description due to D. Luna of all spherical subgroups having spherical closure equal to $\bar{H}$. This is based on the well known combinatorial notion of augmentations and is stated in [Lu01, Proposition 6.4]. However, here we build our discussion on some other auxiliary results from [Lu01, Section 6]: this approach will be useful in Section 7 .

Let us fix a spherically closed subgroup $K$, and consider the following diagram

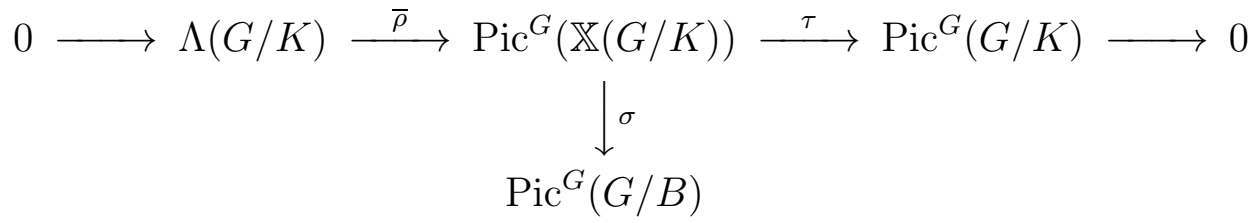

where the row is exact (see also [Br07, Proposition 2.2.1]).

The map $\tau$ is the pullback along the inclusion $G / K \rightarrow \mathbb{X}(G / K)$. For $\sigma$, observe that $\mathbb{X}(G / K)$ has a unique closed $G$-orbit $Z$, which is projective and therefore is equipped with a $G$-equivariant map $G / B \rightarrow Z$. The map $\sigma$ is then the pullback along the composition $G / B \rightarrow Z \rightarrow \mathbb{X}(G / K)$.

The map $\bar{\rho}$ is defined in the following way: for any $\chi \in \Lambda(G / K)$ we pick a function $f_{\chi} \in$ $\mathbb{C}(G / K)_{\chi}^{(B)}$ and consider the $G$-stable part $D=\operatorname{div}\left(f_{\chi}\right)^{G}$ of $\operatorname{div}\left(f_{\chi}\right)$. Then we set $\bar{\rho}(\chi)=$ 
$\mathcal{O}_{\mathbb{X}}(-D)$, with the unique $G$-linearization such that $C$ acts trivially on the total space of the bundle (it exists, since $C$ acts trivially on $\mathbb{X}$ ).

These maps admit also a different interpretation, using the fact that $G=C \times G^{\prime}$ and $K \supseteq C$, that $\Delta(G / K)$ is a basis of $\operatorname{Pic}(\mathbb{X}(G / K))$ (see [Br89, Proposition 2.2]), and the isomorphisms $\operatorname{Pic}^{G}(G / K) \cong \mathcal{X}(K), \operatorname{Pic}^{G}(G / B) \cong \mathcal{X}(B)$. The resulting diagram

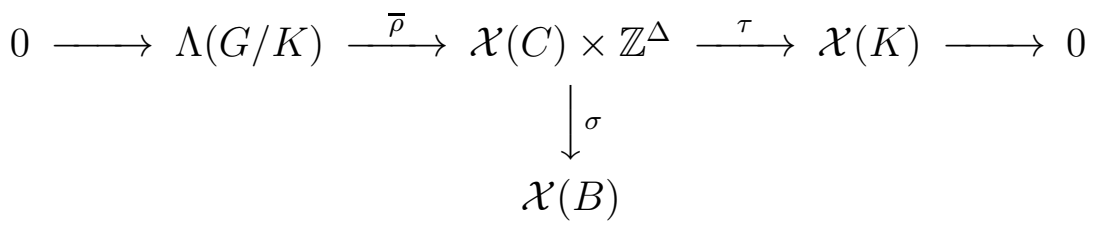

where $\Delta=\Delta(G / K)$, is also described in details in [Lu01, Section 6.3]. As in [Lu01, Lemme 6.2.2], the product $\mathcal{X}(C) \times \mathbb{Z}^{\Delta}$ is identified with the quotient

$$
\frac{\mathbb{C}(G)^{(B \times K)}}{\mathbb{C}^{\times}}
$$

where $B$ acts on $G$ by left translation, $K$ by right translation, and $\mathbb{C}^{\times}$is the multiplicative group of constant non-zero functions on $G$. Under this identification, the map $\tau$ associates to a class $[f]$ the $K$-eigenvalue of $f$, the map $\sigma$ its $B$-eigenvalue, and the map $\bar{\rho}$ associates to $\chi \in \Lambda(G / K)$ the class of a function in $\mathbb{C}(G)_{(\chi, 0)}^{(B \times K)}$. The composition $\sigma \circ \bar{\rho}$ is the identity on $\Lambda(G / K)$, and $\bar{\rho}$ can also be written as

$$
\bar{\rho}(\chi)=\left(\left.\chi\right|_{C},\left\langle\rho_{G / K}(\cdot), \chi\right\rangle\right)
$$

(see loc.cit.).

Lemma 3.7. [Lu01, Lemme 6.3.1, Lemme 6.3.3, Lemme 6.4.1] Let $K \subseteq G$ be a spherically closed subgroup.

(1) The map

$$
H \rightarrow \tau^{-1}\left(\mathcal{X}(K)^{H}\right)
$$

is an inclusion-reversing bijection between the set of normal subgroups $H$ of $K$ such that $K / H$ is diagonalizable, and the set of subgroups of $\mathcal{X}(C) \times \mathbb{Z}^{\Delta}$ containing $\bar{\rho}(\Lambda(G / K))$.

(2) If the restriction of $\sigma$ to $\tau^{-1}\left(\mathcal{X}(K)^{H}\right)$ is injective then $H$ is spherical.

(3) If in addition $\sigma\left(\tau^{-1}\left(\mathcal{X}(K)^{H}\right)\right) \cap \mathrm{S}_{G}^{\circ}(G / K)=\varnothing$ then $\bar{H}=K$, where the set $\mathrm{S}_{G}^{\circ}(G / K)$ is the set of all simple roots $\alpha \in \frac{1}{2} \Sigma(G / K)$ such that $\left\langle\beta^{\vee}, \alpha\right\rangle$ is even for all simple roots $\beta \in \frac{1}{2} \Sigma(G / K)$.

Before showing existence and uniqueness of the wonderful closure, we provide some auxiliary results. They are actually already contained in [Lu01, Section 6], we reprove them here for convenience. 
Lemma 3.8. For any spherical subgroup $H \subseteq G$ contained and normal in another subgroup $K \subseteq G$, and all $D \in \Delta(G / H)$, we have $\pi^{H, K}(D) \in \Delta(G / K)$, and

$$
\pi_{*}^{H, K}\left(\rho_{G / H}(D)\right)=\rho_{G / K}\left(\pi^{H, K}(D)\right) .
$$

Proof. Since $H$ is normal in $K$, then $K$ stabilizes the open set $B H \subseteq G$ acting by right multiplication on $G$ (see [BP87, First part of the proof of Proposition 5.1]). The complement $G \backslash B H=G \backslash B K$ is the union of $\left(\pi^{\left\{e_{G}\right\}, H}\right)^{-1}(E)$ for $E$ varying in $\Delta(G / H)$, and also the union of $\left(\pi^{\left\{e_{G}\right\}, H}\right)^{-1}(F)$ for $F$ varying in $\Delta(G / K)$, whence the first statement.

For the second statement, it is enough to show that a local equation of $D$ on $G / H$ can be chosen to be the pull-back of a function on $G / K$ along $\pi^{H, K}$. Let $E_{1}, \ldots, E_{n}$ be all the distinct $B$-stable prime divisors of $G$ such that $\pi^{\left\{e_{G}\right\}, K}\left(E_{i}\right)=\pi^{H, K}(D)$. Since $G$ is factorial we can choose a global equation $f_{i} \in \mathbb{C}[G]$ for each $E_{i}$, and consider the product $f=f_{1} \cdot \ldots \cdot f_{n}$.

The divisor $\operatorname{div}(f)$ on $G$ is $B$-stable under the left translation action of $G$ on itself, but none of its components is $G$-stable therefore there exists an element $g \in G$ such that the function $f_{0}: x \mapsto f(g x)$ doesn't vanish on any divisor $E_{i}$. On the other hand $\operatorname{div}(f)$ is $K$-stable under the right translation action of $G$ on itself, thus $f$ is $K$-semiinvariant under this action. The function $f_{0}$ is then also $K$-semiinvariant, with same $K$-eigenvalue of $f$. It follows that

$$
F=\frac{f}{f_{0}}
$$

is $K$-invariant with respect to the right translation action. In other words $F=\left(\pi^{\left\{e_{G}\right\}, K}\right)^{*}(\widetilde{F})$ for some $\widetilde{F} \in \mathbb{C}(G / K)$.

Now for some $i_{0}$ the divisor $E_{i_{0}}$ satisfies $\pi^{\left\{e_{G}\right\}, H}\left(E_{i_{0}}\right)=D$. The function $F$ is equal to the pull-back of $\left(\pi^{H, K}\right)^{*}(\widetilde{F})$ along $\pi^{\left\{e_{G}\right\}, H}$ and is a local equation of $E_{i_{0}}$ on $G$, hence $\left(\pi^{H, K}\right)^{*}(\widetilde{F})$ is a local equation of $\pi^{\left\{e_{G}\right\}, H}\left(E_{i_{0}}\right)=D$ on $G / H$ : the lemma follows.

If we suppose in the above lemma that $\bar{H}=K$ then $\Delta=\Delta(G / K)$ and $\Delta(G / H)$ are identified via the map $\pi^{H, K}$ compatibly with the maps $\rho_{G / H}$ and $\rho_{G / K}$. In this case we can extend the $\operatorname{map} \bar{\rho}$ to $\Lambda(G / H)$ as follows.

Definition 3.9. For any $H$ and $K$ as in Lemma 3.8, with $\bar{H}=K$, we denote again by $\bar{\rho}$ the extension of the above map $\bar{\rho}: \Lambda(G / K) \rightarrow \mathcal{X}(C) \times \mathbb{Z}^{\Delta}$ to $\Lambda(G / H)$ defined by:

$$
\bar{\rho}(\chi)=\left(\left.\chi\right|_{C},\left\langle\rho_{G / H}(\cdot), \chi\right\rangle\right) .
$$

Lemma 3.10. For any $H$ and $K$ as in Lemma 3.8 satisfying $\bar{H}=K$, the map $\bar{\rho}: \Lambda(G / H) \rightarrow$ $\mathcal{X}(C) \times \mathbb{Z}^{\Delta}$ is injective and the composition $\sigma \circ \bar{\rho}$ is the identity on $\Lambda(G / H)$. Moreover

$$
\bar{\rho}(\Lambda(G / H))=\tau^{-1}\left(\mathcal{X}(K)^{H}\right) .
$$


Proof. First we observe that

$$
\left(\mathbb{C}(G)^{(B)}\right)^{H} \subseteq \mathbb{C}(G)^{(B \times K)} .
$$

Indeed, let $f \in\left(\mathbb{C}(G)^{(B)}\right)^{H}$. Then $\operatorname{div}(f)$ is $B$-stable under left translation, and $H$-stable under right translation. But $\Delta(G / H)$ and $\Delta(G / K)$ are identified via $\pi^{H, K}$, which implies that $H$ and $K$ act on the set of irreducible components of $G \backslash B H=G \backslash B K$ with the same orbits. Therefore $\operatorname{div}(f)$ is $K$-stable, and $f \in \mathbb{C}(G)^{(B \times K)}$.

It follows that the extended map $\bar{\rho}$ is identified with the inclusion

$$
\Lambda(G / H) \cong \frac{\left(\mathbb{C}(G)^{(B)}\right)^{H}}{\mathbb{C}^{\times}} \subseteq \frac{\mathbb{C}(G)^{(B \times K)}}{\mathbb{C}^{\times}} .
$$

The first two statements follow.

Consider now $\left(\gamma,\left(n_{D}\right)_{D \in \Delta}\right) \in \mathcal{X}(C) \times \mathbb{Z}^{\Delta}$ as the class modulo $\mathbb{C}^{\times}$of a rational function $f \in \mathbb{C}(G)^{(B \times K)}$, with $B$-eigenvalue $\lambda=\sigma\left(\gamma,\left(n_{D}\right)_{D \in \Delta}\right)$ and $K$-eigenvalue $\omega=\tau\left(\gamma,\left(n_{D}\right)_{D \in \Delta}\right)$. Then $\omega$ is trivial on $H$ if and only if $f \in \mathbb{C}(G)^{H}$, if and only if $f$ is the pull-back on $G$ of a function in $\mathbb{C}(G / H)$, if and only if $\lambda \in \Lambda(G / H)$.

Proposition 3.11. Let $H \subseteq G$ be a spherical subgroup, set $K=\bar{H}$ and $\Xi=\operatorname{span}_{\mathbb{Z}} \Sigma(G / H)$. Then

$$
\Lambda(G / K) \subseteq \Xi \subseteq \Lambda(G / H) .
$$

The subgroup $\widehat{H} \subseteq K$ associated with $\bar{\rho}(\Xi)$ via the map of Lemma 3.7 is the unique wonderful closure of $H$. It has the same dimension of $\bar{H}$, and it is the unique wonderful subgroup between $H$ and $\bar{H}$ that satisfies $\Sigma(G / H)=\Sigma(G / \widehat{H})$. Moreover, the spherical closure of $\widehat{H}$ is $\bar{H}$.

Proof. The inclusion $\Xi \subseteq \Lambda(G / H)$ is obvious. The map $\pi_{*}^{H, K}: \mathrm{N}(G / H) \rightarrow \mathrm{N}(G / K)$ has kernel $\mathrm{V}^{\ell}(G / H)$, and satisfies $\pi_{*}(\mathrm{~V}(G / H))=\mathrm{V}(G / K)$. The other inclusion $\Lambda(G / K) \subseteq \Xi$ follows. Hence the subgroup $\widehat{H}$ contains $H$.

The lattice $\Lambda(G / \widehat{H})=\Xi$ has basis $\Sigma(G / H)$ since the spherical roots of a spherical variety are linearly independent. Since $\Lambda(G / \widehat{H})$ has finite index inside $\mathrm{V}^{\ell}(G / H)^{\perp}$ and $\pi^{H, \widehat{H}}(\mathrm{~V}(G / H))=$ $\mathrm{V}(G / \widehat{H})$ we deduce that $\Sigma(G / H)=\Sigma(G / \widehat{H})$.

If $\widetilde{H}$ is another wonderful subgroup such that $H \subseteq \widetilde{H} \subseteq \bar{H}$, then $\Lambda(G / \widetilde{H})$ has also finite index in $\mathrm{V}^{\ell}(G / H)^{\perp}$, and $\Sigma(G / \widetilde{H})$ is equal to $\Sigma(G / H)$ up to taking (positive) multiples of the elements of the latter. The dimension, minimality and uniqueness properties of $\widehat{H}$ follow, since $\Lambda(G / \widetilde{H}) \subseteq \Lambda(G / \widehat{H})$ implies $\widetilde{H} \supseteq \widehat{H}$.

The last assertion follows from part (3) of Lemma 3.7. Indeed, by definition we have $\tau^{-1}\left(\mathcal{X}(K)^{\widehat{H}}\right)=\bar{\rho}(\Xi)$; now the composition $\sigma \circ \bar{\rho}$ is the identity on $\Lambda(G / H)$, which implies that $\sigma\left(\tau^{-1}\left(\mathcal{X}(K)^{\widehat{H}}\right)\right)=\Xi$.

Suppose that $\Xi \cap \mathrm{S}_{G}^{\circ}(G / K) \neq \varnothing$; then there exists a simple root $\alpha \in \Xi$ such that $2 \alpha \in$ $\Sigma(G / K)$. From $\operatorname{ker} \pi_{*}^{H, K}=\mathrm{V}^{\ell}(G / H)$ it also follows that $\alpha \in \Sigma(G / H)$. At this point, after 
[Lu01, Section 1.4], there exist two colors of $G / H$ not stable under $P_{\alpha}$, and there exists only one such color in $G / K$, where $P_{\alpha}$ is the minimal parabolic subgroup of $G$ containing $B$ and associated with $\alpha$. This contradicts the assumption $\bar{H}=K$, therefore $\Xi \cap \mathrm{S}_{G}^{\circ}(G / K)=\varnothing$ and the spherical closure of $\widehat{H}$ is $K$.

\section{Automorphisms stabilizing all $G$-orbits}

From now on, $X$ denotes a complete $G$-regular embedding, with open $G$-orbit $G / H$.

Definition 4.1. For any subset $\mathcal{D} \subseteq \partial_{G} X$ of $G$-stable prime divisors we define

$$
\operatorname{Aut}^{\circ}(X, \mathcal{D})=\left\{\phi \in \operatorname{Aut}^{\circ}(X) \mid \phi(D)=D, \quad \forall D \in \mathcal{D}\right\}
$$

Since $X$ is $G$-regular, the group $\operatorname{Aut}^{\circ}\left(X, \partial_{G} X\right)$ is also the connected group of automorphisms of $X$ stabilizing each $G$-orbit.

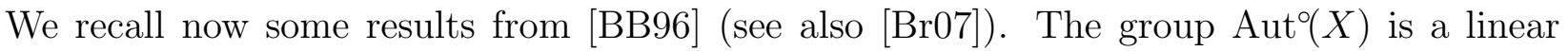
algebraic group, with Lie algebra

$$
\operatorname{Lie} \operatorname{Aut}^{\circ}(X)=H^{0}\left(X, \mathcal{T}_{X}\right)
$$

where $\mathcal{T}_{X}$ is the sheaf of sections of the tangent bundle of $X$. The structure of $G$-module on Lie $\operatorname{Aut}^{\circ}(X)$, induced by the adjoint action of $\theta_{G, X}(G) \subseteq \operatorname{Aut}^{\circ}(X)$, is given in [BB96, Proposition 4.1.1] in terms of global sections of the line bundles $\mathcal{O}_{X}(D)$ where $D \in \partial_{G} X$.

Namely, there exists an exact sequence of $G$-modules

$$
0 \rightarrow \operatorname{Lie} \operatorname{Aut}^{\circ}\left(X, \partial_{G} X\right) \rightarrow \operatorname{Lie} \operatorname{Aut}^{\circ}(X) \rightarrow \bigoplus_{D \in \partial_{G} X} \frac{H^{0}\left(X, \mathcal{O}_{X}(D)\right)}{\mathbb{C}} \rightarrow 0 .
$$

Moreover, for any $\mathcal{D} \in \partial_{G} X$ the Lie algebra of the subgroup $\operatorname{Aut}^{\circ}(X, \mathcal{D})$ is the inverse image of the sum

$$
\bigoplus_{D \in\left(\partial_{G} X\right) \backslash \mathcal{D}} \frac{H^{0}\left(X, \mathcal{O}_{X}(D)\right)}{\mathbb{C}}
$$

We recall that the exact sequence (4.1) is induced by the exact sequence of sheaves

$$
0 \rightarrow \mathcal{S}_{X} \rightarrow \mathcal{T}_{X} \rightarrow \bigoplus_{D \in \partial_{G} X} \mathcal{O}_{X}(D) \otimes_{\mathcal{O}_{X}} \mathcal{O}_{D} \rightarrow 0
$$

Here $\mathcal{S}_{X}$ is the subsheaf of $\mathcal{T}_{X}$ preserving the ideal sheaf of $Y=\bigcup_{D \in \partial_{G} X} D$. We also have that $\mathcal{S}_{X}$ is generated by the image of the map $\mathcal{O}_{X} \otimes \operatorname{Lie} G \rightarrow \mathcal{T}_{X}$ induced by the $G$-action on $X$. The third map in the exact sequence (4.2) can be described locally in a neighborhood of a point $x \in X$ as follows:

$$
\sum_{i=1}^{m} a_{i} \partial_{i} \mapsto \sum_{i}\left(\frac{a_{i}}{x_{i}} \otimes 1\right)
$$


Here we fix a regular system of parameters $x_{1}, \ldots, x_{\operatorname{dim} X}$ such that a local equation of $Y$ is $x_{1}$. $\ldots \cdot x_{m}$. Moreover we denote by $\left(\partial_{1}, \ldots, \partial_{\operatorname{dim} X}\right)$ the dual basis of the local basis $\left(d x_{1}, \ldots, d x_{\operatorname{dim} X}\right)$ of $\Omega_{X}^{1}$ in $x$.

Definition 4.2. Let $0 \neq \gamma \in \mathcal{X}(B)$. If it exists, we denote by $X(\gamma)$ the uniquely determined element of $\partial_{G} X$ such that $H^{0}\left(X, \mathcal{O}_{X}(X(\gamma))\right)_{\gamma}^{(B)} \neq \varnothing$.

A particular case of $\operatorname{Aut}^{\circ}(X)$ has been studied in [Pe09], where $X$ is a wonderful variety. Recall that $C$ acts trivially on any wonderful $G$-variety, hence we can consider $G^{\prime}$-varieties without loss of generality. Moreover, in this case $\operatorname{Aut}^{\circ}(X, \mathcal{D})$ is always semisimple and $X$ is wonderful under its action (see [Br07, Theorem 2.4.2]). It is possible to summarize the results of loc.cit. as follows.

Theorem 4.3. $\left[\mathrm{Pe} 09\right.$ Let $\mathbb{X}$ be a wonderful $G^{\prime}$-variety and $\mathcal{D} \subseteq \partial_{G^{\prime}} \mathbb{X}$. Decompose $G^{\prime}$ and $\mathbb{X}$ into products

$$
G^{\prime}=G_{1}^{\prime} \times \ldots \times G_{n}^{\prime}, \quad \mathbb{X}=\mathbb{X}_{1} \times \ldots \times \mathbb{X}_{n},
$$

with a maximal number of factors in such a way that $G_{i}^{\prime}$ acts non-trivially only on the factor $\mathbb{X}_{i}$ for all $i \in\{1, \ldots, n\}$. Then

$$
\operatorname{Aut}^{\circ}(\mathbb{X}, \mathcal{D})=\operatorname{Aut}^{\circ}\left(\mathbb{X}_{1}, \mathcal{D}_{1}\right) \times \ldots \times \operatorname{Aut}^{\circ}\left(\mathbb{X}_{n}, \mathcal{D}_{n}\right),
$$

where $\mathcal{D}_{i}=\left\{D \cap \mathbb{X}_{i} \mid D \in \mathcal{D}\right\} \subseteq \partial_{G_{i}^{\prime}} \mathbb{X}_{i}$ and $\operatorname{Aut}^{\circ}\left(\mathbb{X}_{i}, \mathcal{D}_{i}\right)$ acts only on $\mathbb{X}_{i}$. Moreover, if the image of $G_{i}^{\prime}$ in $\operatorname{Aut}^{\circ}\left(\mathbb{X}_{i}, \mathcal{D}_{i}\right)$ is a proper subgroup, then $\left(G_{i}^{\prime}, \mathbb{X}_{i}\right)$ appears in the lists of "exceptions" of [Pe09, Sections $3.2-3.6]$. If $\mathcal{D}=\partial_{G^{\prime}} \mathbb{X}$ then all such exceptional factors have rank 0 or 1 , and the rank of $\mathbb{X}$ under the action of $G^{\prime}$ and of $\operatorname{Aut}^{\circ}\left(\mathbb{X}, \partial_{G^{\prime}} \mathbb{X}\right)$ coincide.

The invariants of $\mathbb{X}_{i}$ under the action of $\operatorname{Aut}^{\circ}\left(\mathbb{X}_{i}, \mathcal{D}_{i}\right)$ are also listed in loc.cit., for all cases where the image of $G_{i}^{\prime}$ in $\operatorname{Aut}^{\circ}\left(\mathbb{X}_{i}, \mathcal{D}_{i}\right)$ is a proper subgroup.

Now, the group $\operatorname{Aut}^{\circ}\left(X, \partial_{G} X\right)$ has been described in [Br07]. Namely, thanks to [Br07, Theorem 4.4.1, part (1)], there exists a split exact sequence of Lie algebras

$$
0 \rightarrow \frac{\operatorname{Lie} \bar{H}}{\operatorname{Lie} H} \rightarrow \operatorname{Lie}_{A_{u t}}\left(X, \partial_{G} X\right) \rightarrow \operatorname{Lie} \operatorname{Aut}^{\circ}\left(\mathbb{X}(G / \bar{H}), \partial_{G} \mathbb{X}(G / \bar{H})\right) \rightarrow 0 .
$$

The same holds if we consider the variety $\mathbb{X}(G / \widehat{H})$ instead of $\mathbb{X}(G / \bar{H})$. Indeed, the results of [Br07, Section 4.4] hold (with same proofs) if we replace the spherical closure of $H$ with its wonderful closure.

It follows that $\operatorname{Aut}^{\circ}\left(X, \partial_{G} X\right)$ is reductive, its connected center is $(\bar{H} / H)^{\circ}=(\widehat{H} / H)^{\circ}$, and its semisimple part can be computed using Theorem 4.3 and the lists of [Pe09].

The invariants of $X$ under the action of $\operatorname{Aut}^{\circ}\left(X, \partial_{G} X\right)$ are easily recovered. Namely, if we choose a Borel subgroup of $\operatorname{Aut}^{\circ}\left(X, \partial_{G} X\right)$ containing the image of $B$, then colors (see $\operatorname{Br} 07$, 
Theorem 4.4.1, part (2)]) and boundary divisors are the same of the $G$-action, which also induces a natural identification of $\Lambda_{G}(X)$ and $\Lambda_{\mathrm{Aut}\left(X, \partial_{G} X\right)}(X)$.

The spherical roots for the $\operatorname{Aut}^{\circ}\left(X, \partial_{G} X\right)$-action and the parabolic subgroup $\mathrm{P}_{\mathrm{Aut}^{\circ}\left(X, \partial_{G} X\right)}(X)$ are the same as those of $\mathbb{X}(G / \widehat{H})$; for the factors of $G$ that do not map surjectively onto factors of $\operatorname{Aut}^{\circ}\left(X, \partial_{G} X\right)$ these invariants are computed in [Pe09, Sections 3.2-3.3].

Finally, the structure of the orbits of $\operatorname{Aut}^{\circ}\left(X, \partial_{G} X\right)$ and of $G$ on $X$ coincide, which implies the equality of the fans of polyhedral convex cones of $X$ with respect to the two actions.

Example 4.4. Let $V$ be the irreducible 8-dimensional Spin(7)-module. Consider $G=\mathbb{G}_{m} \times$ $\operatorname{Spin}(7)$ acting on $X=\mathrm{Bl}_{[0,1]}(\mathbb{P}(V \oplus \mathbb{C}))$. Then $X$ is a regular $G$-variety, with one $G$-color (corresponding to an hyperplane in $V$ ) and two $G$-stable prime divisors (one given by $V$ itself, the other corresponding to the cone in $V$ over a smooth quadric in $\mathbb{P}(V))$. We have $\Sigma_{G}(X)=$ $\left\{\alpha_{1}+2 \alpha_{2}+3 \alpha_{3}\right\}$ and $\mathrm{S}_{G}^{p}(X)=\left\{\alpha_{1}, \alpha_{2}\right\}$. The groups $\bar{H}$ and $\widehat{H}$ are equal, the variety $\mathbb{X}=$ $\mathbb{X}(G / \bar{H})$ is $\mathbb{P}(V)=\mathbb{P}^{7}$ and $G$ acts on it via its quotient $\operatorname{PSO}(7)$. It occurs in [Pe09, Section $3.3]$; it has one $G$-color and only one $G$-stable prime divisor.

Notice that the natural map between the open $G$-orbits extends to a map $X \rightarrow \mathbb{X}$, and that taking inverse images does not induce a bijection between $\partial \mathbb{X}$ and $\partial X$. Nevertheless the automorphism groups stabilizing the whole boundary correspond, up to a torus factor and up to central isogeny: we have $\operatorname{Aut}^{\circ}(X, \partial X)=\mathbb{G}_{m} \times \mathrm{SO}(8)$ and $\operatorname{Aut}^{\circ}(\mathbb{X}, \partial \mathbb{X})=\operatorname{PSO}(8)$. The other invariants behave as above: the $\operatorname{Aut}^{\circ}(X, \partial X)$-colors and $\operatorname{Aut}^{\circ}(X, \partial X)$-stable prime divisors are the same of the $G$-action, and the same happens for $\mathbb{X}$. Finally, we have $\Sigma_{\mathrm{Aut}{ }^{\circ}(X, \partial X)}(X)=$ $\Sigma_{\mathrm{Aut}^{\circ}(\mathbb{X}, \partial \mathbb{X})}(\mathbb{X})=\left\{2 \alpha_{1}+2 \alpha_{2}+\alpha_{3}+\alpha_{4}\right\}$ and $\mathrm{S}_{\mathrm{Aut}(X, \partial X)}^{p}(X)=\mathrm{S}_{\mathrm{Aut}(\mathbb{X}, \partial \mathbb{X})}^{p}(\mathbb{X})=\left\{\alpha_{1}, \alpha_{2}\right\}$

\section{5. $\operatorname{Relating} \operatorname{Aut}^{\circ}(X)$ To $\operatorname{Aut}^{\circ}(\mathbb{X})$}

From now on, $\mathbb{X}=\mathbb{X}(G / \widehat{H})$ denotes the wonderful embedding of $G / \widehat{H}$. As a consequence of the last section, we assume that $\theta_{G, X}(G)=\operatorname{Aut}^{\circ}\left(X, \partial_{G} X\right)$ and that $\theta_{G, \mathbb{X}}(G)=\operatorname{Aut}^{\circ}\left(\mathbb{X}, \partial_{G} \mathbb{X}\right)$. Indeed, if this is not the case we may first apply Theorem 4.3 to $\mathbb{X}$, replace $G_{i}^{\prime}$ with the universal cover of $\operatorname{Aut}^{\circ}\left(\mathbb{X}_{i}, \partial_{G_{i}} \mathbb{X}_{i}\right)$ for all $i$ such that these groups are different, and then replace $C$ with $C \times(\widehat{H} / H)^{\circ}$.

Lemma 5.1. The map $\pi^{H, \widehat{H}}: G / H \rightarrow G / \widehat{H}$ extends to a surjective $G$-equivariant map $\pi: X \rightarrow$ $\mathbb{X}$. An element $D \in \partial_{G} X$ is mapped surjectively onto $\mathbb{X}$ if and only if $D \in\left(\partial_{G} X\right)^{\ell}$.

Proof. The fan $\mathcal{F}_{G}(\mathbb{X})$ contains the unique maximal cone $\mathrm{V}_{G}(\mathbb{X})$. Then, since $X$ is toroidal, the extended map $\pi: X \rightarrow \mathbb{X}$ exists by [Kn96, Theorem 4.1].

The condition $\pi(D)=\mathbb{X}$ is equivalent to the condition that $\pi(Y)=G / \widehat{H}$, where $Y \subseteq X$ is the union of $G / H$ and the open $G$-orbit of $D$. This is also equivalent to the fact that the map $\pi^{H, \widehat{H}}$ extends to $Y \rightarrow G / \widehat{H}$. 
The fan $\mathcal{F}_{G}(Y)$ has only one maximal cone, namely $\mathbb{Q}_{\geq 0} \rho_{G, X}(D)$, and the fan $\mathcal{F}_{G}(G / \widehat{H}$ has only the trivial cone $\{0\}$. Then, thanks to [Kn96, Theorem 4.1], the map $\pi^{H, \widehat{H}}$ extends to $Y \rightarrow G / \widehat{H}$ if and only if $\rho_{G, X}(D)$ is in the kernel of ker $\pi_{*}^{H, \widehat{H}}$, which is $\mathrm{V}^{\ell}(G / H)$.

Example 5.2. Let $G=\mathrm{SL}(n+1)$ and $X=\mathrm{Bl}_{p}\left(\mathbb{P}^{n+1}\right) \times\left(\mathbb{P}^{n}\right)^{*}$ as in Example 2.6. We have $\mathbb{X}=\mathbb{P}^{n} \times\left(\mathbb{P}^{n}\right)^{*}$, and the image $\pi(E) \subset \mathbb{X}$ is the subset $\{([v],[\eta]) \mid \eta(v)=0\}$, i.e. the unique $G$-stable prime divisor of $\mathbb{X}$. The two other $G$-stable prime divisors of $X$ are both mapped surjectively onto $\mathbb{X}$.

Definition 5.3. We denote by

$$
X \stackrel{\psi}{\longrightarrow} X^{\prime} \stackrel{f}{\longrightarrow} \mathbb{X}
$$

the Stein factorization of the map $\pi: X \rightarrow \mathbb{X}$.

In [Br07, Section 4.4] it is shown that $\operatorname{Aut}^{\circ}(X)$ acts on $X^{\prime}$ in such a way that $\psi$ is equivariant; we denote the corresponding homomorphism as follows:

$$
\psi_{*}: \operatorname{Aut}^{\circ}(X) \rightarrow \operatorname{Aut}^{\circ}\left(X^{\prime}\right) .
$$

Its kernel is the subgroup of automorphisms of $X$ stabilizing each fiber of $\psi$.

Proposition 5.4. The inclusions $\mathrm{Z}\left(\theta_{G, X}(G)\right)^{\circ} \subseteq \operatorname{ker} \psi_{*} \cap \theta_{G, X}(G) \subseteq \mathrm{Z}\left(\theta_{G, X}(G)\right)$ between subgroups of $\operatorname{Aut}^{\circ}(X)$ hold. Moreover, there is a local isomorphism

$$
\operatorname{Aut}^{\circ}\left(X,\left(\partial_{G} X\right)^{n \ell}\right) \cong \theta_{G, X}\left(G^{\prime}\right) \ltimes\left(\operatorname{ker} \psi_{*}\right)^{\circ}
$$

induced by the inclusion of both factors of the right hand side in $\operatorname{Aut}^{\circ}(X)$.

Proof. The first inclusion stems from the fact that $C=\mathrm{Z}(G)^{\circ}$ acts trivially on $\mathbb{X}$, hence also on $X^{\prime}$. On the other hand, if $g \in G$ stabilizes all fibers of $\psi$, then it acts trivially on $X^{\prime}$ and also on $\mathbb{X}$. Therefore, to show the second inclusion, we only have to check that no simple factor of $G$ acts trivially on $\mathbb{X}$ but not on $X$. This is true because $\widehat{H} / H$ is abelian.

Let us prove the last statement. Both groups on the right hand side of (5.1) are subgroups of $\operatorname{Aut}^{\circ}\left(X,\left(\partial_{G} X\right)^{n \ell}\right)$ : this is obvious for $\theta_{G, X}\left(G^{\prime}\right)$, so we only have to check it for $\left(\operatorname{ker} \psi_{*}\right)^{\circ}$. Notice that $\psi$ maps any element $D$ of $\left(\partial_{G} X\right)^{n \ell}$ onto a proper $G$-stable closed subset of $X^{\prime}$ by Lemma 5.1. It follows that $D$ is an irreducible component of $\psi^{-1}(\psi(D))$, hence it is stable under the action of $\left(\operatorname{ker} \psi_{*}\right)^{\circ}$. It also follows that $\psi_{*}$ maps $\operatorname{Aut}^{\circ}\left(X,\left(\partial_{G} X\right)^{n \ell}\right) \operatorname{into} \operatorname{Aut}^{\circ}\left(X^{\prime}, \partial_{G} X^{\prime}\right)$.

The intersection $\left(\operatorname{ker} \psi_{*}\right)^{\circ} \cap \theta_{G, X}\left(G^{\prime}\right)$ is finite thanks to the first part of the proof, and $\left(\operatorname{ker} \psi_{*}\right)^{\circ}$ is a normal subgroup of $\operatorname{Aut}^{\circ}\left(X,\left(\partial_{G} X\right)^{n \ell}\right)$. It only remains to prove that $\operatorname{Aut}^{\circ}\left(X,\left(\partial_{G} X\right)^{n \ell}\right)$ is generated by $\theta_{G, X}\left(G^{\prime}\right)$ and $\left(\operatorname{ker} \psi_{*}\right)^{\circ}$.

By [Br07, Theorem 4.4.1], we know that $\operatorname{Aut}^{\circ}\left(X^{\prime}, \partial X^{\prime}\right)$ and $\operatorname{Aut}^{\circ}(\mathbb{X}, \partial \mathbb{X})$ are both semisimple and locally isomorphic. It follows that the universal cover of $\operatorname{Aut}^{\circ}\left(X^{\prime}, \partial X^{\prime}\right)$ acts on $\mathbb{X}$ in such 
a way that $f$ is equivariant. On the other hand no element of this universal cover could act trivially on $X^{\prime}$ and non-trivially on $\mathbb{X}$, hence $\operatorname{Aut}^{\circ}\left(X^{\prime}, \partial X^{\prime}\right)$ itself acts on $\mathbb{X}$, preserving all $G$-orbits. This produces a commutative diagram

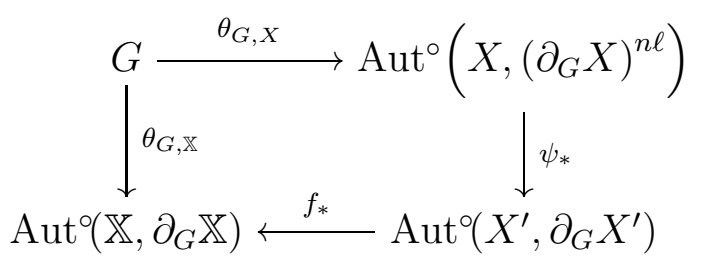

where $\theta_{G, \mathbb{X}}$ is surjective by our assumptions. Therefore $\operatorname{Aut}^{\circ}\left(X,\left(\partial_{G} X\right)^{n \ell}\right)$ is generated by $\theta_{G, X}(G)$ and $\operatorname{ker}\left(f_{*} \circ \psi_{*}\right)$. Notice that $f_{*}$ has finite kernel, that the kernel of $\psi_{*}$ contains $\theta_{G, X}(C)$, and that $\operatorname{Aut}^{\circ}\left(X,\left(\partial_{G} X\right)^{n \ell}\right)$ is connected: we deduce that $\operatorname{Aut}^{\circ}\left(X,\left(\partial_{G} X\right)^{n \ell}\right)$ is indeed generated by $\theta_{G, X}\left(G^{\prime}\right)$ and $\left(\operatorname{ker} \psi_{*}\right)^{\circ}$, and the proof is complete.

If we denote by

$$
d \psi_{*}: \operatorname{Lie}_{\operatorname{Aut}^{\circ}}(X) \rightarrow \operatorname{Lie}_{\operatorname{Aut}^{\circ}\left(X^{\prime}\right)}
$$

the corresponding homomorphism of Lie algebras, then the following corollary is an immediate consequence of the above proposition.

Corollary 5.5. The subspace ker $d \psi_{*} \subseteq$ Lie $\operatorname{Aut}^{\circ}(X)$ is $G$-stable, and its intersection with Lie $\theta_{G, X}(G)$ is equal to Lie $\theta_{G, X}(C)$. There exists a $G$-equivariant splitting of the exact sequence (4.1) such that

$$
\operatorname{ker} d \psi_{*}=\operatorname{Lie} \theta_{G, X}(C) \oplus \bigoplus_{D \in\left(\partial_{G} X\right)^{\ell}} \frac{H^{0}\left(X, \mathcal{O}_{X}(D)\right)}{\mathbb{C}}
$$

\section{Restricting Automorphisms of $X$ to Fibers of $\psi$}

We study now the automorphisms of a generic fiber of $\psi$ induced by automorphisms of $X$ belonging to ker $\psi_{*}$. For this it is convenient to exploit the local structure of spherical varieties.

Theorem 6.1. Kn94, Theorem 2.3 and Proposition 2.4] Let $Y$ be a spherical G-variety. Let $P_{G, Y} \supseteq B$ be the stabilizer in $G$ of the open $B$-orbit of $Y$, let $L_{G, Y}$ be the Levi subgroup of $P_{G, Y}$ containing $T$, and consider the following open subset of $Y$ :

$$
Y_{0}=Y \backslash \bigcup_{D \in \Delta_{G}(Y)} D
$$

Then there exists a closed $L_{G, Y}$-stable and $L_{G, Y}$-spherical subvariety $\mathcal{Z}_{G, Y}$ of $Y_{0}$ such that the map

$$
\begin{array}{cl}
P_{G, Y}^{u} \times \mathcal{Z}_{G, Y} & \rightarrow Y_{0} \\
(p, z) & \mapsto p z
\end{array}
$$


is a $P_{G, Y}$-equivariant isomorphism, where $L_{G, Y}$ acts on $P_{G, Y}^{u} \times \mathcal{Z}_{G, Y}$ by $l \cdot(p, z)=\left(l p l^{-1}, l z\right)$. The commutator subgroup $\left(L_{G, Y}, L_{G, Y}\right)$ acts trivially on $\mathcal{Z}_{G, Y}$, and if $Y$ is toroidal then every $G$-orbit meets $\mathcal{Z}_{G, Y}$ in an $L_{G, Y}$-orbit.

Definition 6.2. We define $T_{G, Y}$ to be the quotient of $L_{G, Y}^{r}$ by the kernel of its action on $\mathcal{Z}_{G, Y}$.

We get back to our complete $G$-regular embedding $X$. The torus $T_{G, X}$ is a subquotient of $T$, and $\mathcal{Z}_{G, X}$ is a spherical (toric) $T_{G, X}$-variety, with lattice $\Lambda_{T_{G, X}}\left(\mathcal{Z}_{G, X}\right)=\mathcal{X}\left(T_{G, X}\right)=\Lambda_{G}(G / H)$ and fan of convex cones equal to $\mathcal{F}_{G}(X)$.

Definition 6.3. For any $x^{\prime}$ in the open $G$-orbit of $X^{\prime}$ we denote by $\kappa_{x^{\prime}}$ the restriction map

$$
\kappa_{x^{\prime}}:\left(\operatorname{ker} \psi_{*}\right)^{\circ} \rightarrow \operatorname{Aut}^{\circ}\left(X_{x^{\prime}}\right)
$$

where $X_{x^{\prime}}=\psi^{-1}\left(x^{\prime}\right)$.

Recall that $H$ is chosen in such a way that $B H$ is open in $G$, and $x_{0}=e H \in G / H \subseteq X$. Let us consider $x_{0}^{\prime}=\psi\left(x_{0}\right)$ : the fiber $X_{x_{0}^{\prime}}$ is smooth and complete, and it is a toric variety under the action of the torus $S=(\widehat{H} / H)^{\circ}=H^{\prime} / H$, where $H^{\prime}$ is the stabilizer of $x_{0}^{\prime}$.

Moreover, $S$ acts naturally on $G / H$ by $G$-equivariant automorphisms, and since $S$ is connected this $S$-action extends to $X$, stabilizing all colors of $X$ and all fibers of $\psi$. We may fix $\mathcal{Z}_{G, X^{\prime}} \subset X^{\prime}$ containing $x_{0}^{\prime}$, and choose $\mathcal{Z}_{G, X}$ so that

$$
\mathcal{Z}_{G, X}=\psi^{-1}\left(\mathcal{Z}_{G, X^{\prime}}\right) \cap X_{0},
$$

which implies that $\mathcal{Z}_{G, X}$ contains $x_{0}$ and is stable under the action of $S$.

Lemma 6.4. The above action of $S$ on $\mathcal{Z}_{G, X}$ can be realized sending $S$ injectively into $T_{G, X}$, and then letting it act on $\mathcal{Z}_{G, X}$ via the restriction of the usual action of $G$ on $X$.

Proof. If $n H \in S$ and $f \in \mathbb{C}(G / H)_{\chi}^{(B)}$, then $g H \mapsto f(g n H)$ also belongs to $\mathbb{C}(G / H)_{\chi}^{(B)}$, therefore there is a homomorphism (depending only on $\chi$ ) $\iota_{\chi}: S \rightarrow \mathbb{G}_{m}$ such that $f(g n H)=$ $\iota_{\chi}\left(n^{-1} H\right) f(g H)$ for all $g \in G$. This induces a homomorphism

$$
\iota: S \rightarrow \operatorname{Hom}\left(\Lambda_{G}(G / H), \mathbb{G}_{m}\right) \cong T_{G, X},
$$

which can be shown to be injective, with image equal to the subtorus of $T_{G, X}$ corresponding to the subspace $\mathrm{V}_{G}^{\ell}(G / H) \subseteq \mathrm{N}_{G}(G / H)$ (see [Br97, Proof of Theorem 4.3]). Let us check that the restriction of the usual $T_{G, X}$-action on $\mathcal{Z}_{G, X}$ to the subtorus $\iota(S)$ yields the action described above. The intersection $\mathcal{Z}_{G, X} \cap G / H$ is dense in $\mathcal{Z}_{G, X}$, and $\mathcal{Z}_{G, X}$ is a toric $T_{G, X}$-variety with lattice equal to $\Lambda_{G}(G / H)$ : it follows that $\iota(n H) g H=g n H$, because

$$
f(\iota(n H) g H)=\chi\left(\iota\left(n^{-1} H\right)\right) f(g H)=\iota_{\chi}\left(n^{-1} H\right) f(g H)=f(g n H)
$$

for all $n H \in S, g H \in \mathcal{Z}_{G, X} \cap G / H, \chi \in \Lambda_{G}(G / H)$ and $f \in \mathbb{C}(G / H)_{\chi}^{(B)}$. 
The fiber $X_{x_{0}^{\prime}}$ is also the fiber over $x_{0}^{\prime}$ of the $S$-equivariant map $\mathcal{Z}_{G, X} \rightarrow \mathcal{Z}_{G, X^{\prime}}$, which implies that its fan of convex cones is

$$
\mathcal{F}_{S}\left(X_{x_{0}^{\prime}}\right)=\left\{c \mid c \in \mathcal{F}_{G}(X), c \subset \mathrm{V}_{G}^{\ell}(G / H)\right\} .
$$

Since the $S$-boundary of $X_{x_{0}^{\prime}}$ is given intersecting $X_{x_{0}^{\prime}}$ with the elements of $\left(\partial_{G} X\right)^{\ell}$, there is an exact sequence of $S$-modules

$$
0 \rightarrow \operatorname{Lie} S \rightarrow \operatorname{Lie} \operatorname{Aut}^{\circ}\left(X_{x_{0}^{\prime}}\right) \rightarrow \bigoplus_{D \in\left(\partial_{G} X\right)^{\ell}} \frac{H^{0}\left(X_{x_{0}^{\prime}}, \mathcal{O}_{X}\left(D \cap X_{x_{0}^{\prime}}\right)\right)}{\mathbb{C}} \rightarrow 0
$$

Lemma 6.5. Consider the homomorphism

$$
d \kappa_{x^{\prime}}: \operatorname{ker} d \psi_{*}=\operatorname{Lie}\left(\operatorname{ker} \psi_{*}\right)^{\circ} \rightarrow \operatorname{Lie} \operatorname{Aut}^{\circ}\left(X_{x^{\prime}}\right)
$$

where $x^{\prime}$ is in the open B-orbit of $X^{\prime}$. If $V \subseteq \operatorname{ker} d \psi_{*}$ is a simple $G$-submodule, then $d \kappa_{x^{\prime}}(V)=$ $d \kappa_{x^{\prime}}(\mathbb{C} v)$, where $v \in V$ is a highest weight vector.

Proof. We may assume that $x^{\prime}=x_{0}^{\prime}$ and that $v=[s] \in H^{0}\left(X, \mathcal{O}_{X}(D)\right) / \mathbb{C}$ for some $D \in(\partial X)^{\ell}$, in view of Corollary 5.5. From the expression in local coordinates (4.3), it follows that $d \kappa_{x_{0}^{\prime}}(v)$

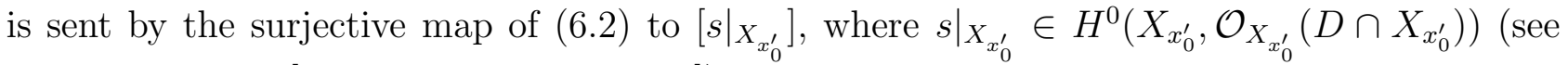
also the proof of [BB96, Proposition 4.1.1]).

If $s$ is a $B$-eigenvector then its zeros are $B$-stable. On the other hand, since $B x_{0}^{\prime}$ is open in $X^{\prime}$, the only zeros of $s$ intersecting $X_{x_{0}^{\prime}}$ are $G$-stable. It also follows that $\left.(g s)\right|_{X_{x_{0}^{\prime}}}$ and $\left.s\right|_{X_{x_{0}^{\prime}}}$ have the same zeros (hence are linearly dependent) for any $g \in G$ such that $g x_{0}$ doesn't lie on any color of $G / H$. This is true for $g$ lying in the dense subset $B H$ of $G$, and since $V$ is generated as a vector space by elements of the form $[g s]$ for $g \in B H$, the lemma follows.

The following lemma on the structure of $\mathcal{F}_{G}(X)$ will be crucial in the study of the image of $\kappa_{x^{\prime}}$.

Lemma 6.6. Let $G$ be Let $i=1,2$ and $0 \neq \gamma_{i} \in \Lambda_{G}(X)$ be such that $X\left(\gamma_{i}\right)$ exists, with $X\left(\gamma_{i}\right) \in(\partial X)^{\ell}$. Suppose that $\left\langle m, \gamma_{1}\right\rangle=-\left\langle m, \gamma_{2}\right\rangle$ for all $m \in \mathrm{V}_{G}^{\ell}(X)$. Then

$$
\left\langle\rho_{G, X}(D), \gamma_{i}\right\rangle=0
$$

for all $i=1,2$, for all $D \in\left(\partial_{G} X\right)^{n \ell}$ and for all $D \in \Delta_{G}(X)$.

Proof. Consider the wonderful variety $\mathbb{X}$. Both sets $\rho_{G, \mathbb{X}}\left(\Delta_{G}(\mathbb{X})\right)$ and $\rho_{G, \mathbb{X}}\left(\partial_{G} \mathbb{X}\right)$ generate $\mathrm{N}_{G}(\mathbb{X})$ as a vector space, and the convex cone generated by $\rho_{G, \mathbb{X}}\left(\Delta_{G}(\mathbb{X})\right)$ contains $-\rho_{G, \mathbb{X}}\left(\partial_{G} \mathbb{X}\right)($ see [Br07, Lemma 2.1.2]). On the other hand, the set $\pi_{*}\left(\rho_{G, X}\left(\left(\partial_{G} X\right)^{n \ell}\right)\right) \subset \mathrm{N}_{G}(\mathbb{X})$ generates the same convex cone $\mathrm{V}_{G}(\mathbb{X})$ generated by $\rho_{G, \mathbb{X}}\left(\partial_{G} \mathbb{X}\right)$, and $\pi_{*}\left(\rho_{G, X}\left(\Delta_{G}(X)\right)\right)=\rho_{G, \mathbb{X}}\left(\Delta_{G}(\mathbb{X})\right)$. It follows that there exists a linear combination

$$
v=\sum_{Y \in\left(\partial_{G} X\right)^{n \ell}} n_{Y} \rho_{G, X}(Y)+\sum_{Z \in \Delta_{G}(X)} n_{Z} \rho_{G, X}(Z) \in \mathrm{V}_{G}^{\ell}(X)
$$


where all the coefficients $n_{Y}$ and $n_{Z}$ are positive. From the assumptions on the characters $\gamma_{i}$, all the elements $\rho_{G, X}(Y)$ and $\rho_{G, X}(Z)$ above are non-negative on both $\gamma_{1}$ and $\gamma_{2}$ : we deduce that $\left\langle v, \gamma_{i}\right\rangle \geq 0$, which yields $\left\langle v, \gamma_{i}\right\rangle=0$. The lemma follows.

\section{PRime Divisors not on the Linear PART OF THE VALUATion CONE}

In this section we study $\operatorname{Aut}^{\circ}(X, \mathcal{D})$ under the assumption that $\mathcal{D} \supseteq\left(\partial_{G} X\right)^{\ell}$. We also suppose that $\mathcal{D}$ contains all the $G$-stable prime divisors $D$ that satisfy $H^{0}\left(X, \mathcal{O}_{X}(D)\right)=\mathbb{C}$, since these prime divisors do not move under the action of $\operatorname{Aut}^{\circ}(X)$ anyway, and define $\mathcal{E}=\partial X \backslash \mathcal{D}$.

Before stating the main result of this section, Theorem 7.9, we need to establish a correspondence between the divisors in $\mathcal{E}$ and certain boundary divisors of $\mathbb{X}$.

Recall that, since $\mathbb{X}$ is wonderful, the set $-\rho_{G, \mathbb{X}}\left(\partial_{X} \mathbb{X}\right)$ is a basis of $\mathrm{N}_{G}(\mathbb{X})$, dual to $\Sigma_{G}(\mathbb{X})$.

Definition 7.1. For an element $D \in \partial_{G} \mathbb{X}$, we denote by $\sigma_{D}$ the spherical root of $\mathbb{X}$ dual to $-\rho_{G, \mathbb{X}}(D)$.

Since $\Lambda_{G}(\mathbb{X})$ is a sublattice of $\Lambda_{G}(X)$, we consider $\sigma_{D}$ also as an element of the latter. Also recall that, thanks to $\left[\mathrm{Br} 07\right.$, Theorem 2.2.3], if $D \in \partial_{G} \mathbb{X}$ satisfies $H^{0}\left(\mathbb{X}, \mathcal{O}_{\mathbb{X}}(D)\right) \neq \mathbb{C}$ then $H^{0}\left(\mathbb{X}, \mathcal{O}_{\mathbb{X}}(D)\right) / \mathbb{C}$ is irreducible with highest weight $\sigma_{D}$.

Lemma 7.2. Let $E \in \mathcal{E}$. Then:

(1) the image $\pi(E)$ is an element of $\partial_{G} \mathbb{X}$, with $H^{0}\left(\mathbb{X}, \mathcal{O}_{\mathbb{X}}(\pi(E))\right) \neq \mathbb{C}$, and $E$ is the only element of $\partial_{G} X$ whose image is $\pi(E)$;

(2) we have

$$
\pi_{*}\left(\rho_{G, X}(E)\right)=\rho_{G, \mathbb{X}}(\pi(E)),
$$

and

$$
\forall c \in \mathcal{F}_{G}(X) \backslash\left\{c_{X, E}\right\}, c 1 \text {-dimensional: } \quad c \subset \sigma_{\pi(E)}^{\perp} ;
$$

(3) the $G$-modules $H^{0}\left(X, \mathcal{O}_{X}(E)\right)$ and $H^{0}\left(\mathbb{X}, \mathcal{O}_{\mathbb{X}}(\pi(E))\right)$ are isomorphic.

Proof. Let $\gamma \neq 0$ be such that $H^{0}\left(X, \mathcal{O}_{X}(E)\right)_{\gamma}^{(B)} \neq \varnothing$. Since $E \in\left(\partial_{G} X\right)^{n \ell}$, the character $\gamma$ is non-negative on $\rho_{G, X}\left(\left(\partial_{G} X\right)^{\ell}\right)$, which generates the whole $\mathrm{V}_{G}^{\ell}(X)$ as a convex cone, because $X$ is complete. It follows that $\gamma \in \mathrm{V}_{G}^{\ell}(X)^{\perp}$, which implies that some positive integral multiple of $\gamma$, say $n \gamma$, lies in $\Lambda_{G}(\mathbb{X})$. Let us also assume that it is indecomposable in $\Lambda_{G}(\mathbb{X})$, i.e. that $n$ is minimal satisfying $n>0$ and $n \gamma \in \Lambda_{G}(X)$.

Consider $\pi(E)$ : it is a proper subset of $\mathbb{X}$ because $\pi_{*}\left(\rho_{G, X}(E)\right) \neq 0$. If it is not a $G$-stable prime divisor of $\mathbb{X}$, then $\pi_{*}\left(\rho_{G, X}(E)\right)$ doesn't lie on any 1-dimensional face of $\mathrm{V}_{G}(\mathbb{X})$. On the other hand, each element of $\partial_{G} \mathbb{X}$ is the image $\pi(D)$ of some $G$-stable prime divisor $D$ of $X$, with $\pi_{*}\left(\rho_{G, X}(D)\right)$ equal to a positive rational multiple of $\rho_{G, \mathbb{X}}(\pi(D))$. This implies that $n \gamma \in \Lambda_{G}(\mathbb{X})$ 
is non-negative on $\rho_{G, \mathbb{X}}\left(\partial_{G} \mathbb{X}\right)$ and negative on $\pi_{*}\left(\rho_{G, X}(E)\right)$, which is absurd because $\rho_{G, \mathbb{X}}\left(\partial_{G} \mathbb{X}\right)$ generates $\mathrm{V}_{G}(\mathbb{X})$ as a convex cone.

We conclude that $\pi(E) \in \partial_{G} \mathbb{X}$, and that $E$ is the unique element of $\partial_{G} X$ whose image is $\pi(E)$, because $n \gamma$ is non-negative on $\rho_{G, X}\left(E^{\prime}\right)$ for any $E^{\prime} \in \partial_{G} X$ different from $E$. Let $0>-m=\left\langle\rho_{G, \mathbb{X}}(\pi(E)), n \gamma\right\rangle$. Then $H^{0}\left(\mathbb{X}, \mathcal{O}_{\mathbb{X}}(m \pi(E))\right) \neq \mathbb{C}$.

From [Br07, Theorem 2.2.3] it follows that $H^{0}\left(\mathbb{X}, \mathcal{O}_{\mathbb{X}}(\pi(E))\right) \neq \mathbb{C}$, that the quotient $H^{0}\left(\mathbb{X}, \mathcal{O}_{\mathbb{X}}(\pi(E))\right) / \mathbb{C}$ is irreducible with highest weight $\sigma_{\pi(E)}$, and that any $\chi \in \Lambda_{G}(\mathbb{X})$ satisfying

$$
\left\langle\rho_{G, \mathbb{X}}(D), \chi\right\rangle \geq 0 \quad \forall D \in\left(\partial_{G} \mathbb{X} \backslash\{\pi(E)\} \cup \Delta_{G}(\mathbb{X}), \quad\left\langle\rho_{G, \mathbb{X}}(\pi(E)), \chi\right\rangle<0\right.
$$

is a positive multiple of $\sigma_{\pi(E)}$. We have then shown (11). It also follows that $n \gamma$ is a positive multiple of $\sigma_{\pi(E)}$, whence $\gamma$ is non-positive on $\mathrm{V}_{G}(X)$ and so it is zero on $\rho_{G, X}(D)$ for all $D \in \partial_{G} X$ different from $E$. This shows (7.1).

Now recall that $n \gamma$ is indecomposable in $\Lambda_{G}(\mathbb{X})$. Since it is a positive multiple of $\sigma_{\pi(E)}$, it is equal to $\sigma_{\pi(E)}$. On the other hand $\Sigma_{G}(X)=\Sigma_{G}(\mathbb{X})$ and $\sigma_{\pi(E)}$ is also indecomposable in $\Lambda_{G}(X)$. Therefore $n=1$, and we have

$$
\left\langle\rho_{G, X}(E), \gamma\right\rangle=\left\langle\pi_{*}\left(\rho_{G, X}(E)\right), \gamma\right\rangle=-1=\left\langle\rho_{G, \mathbb{X}}(\pi(E)), \sigma_{\pi(E)}\right\rangle,
$$

whence $\pi_{*}\left(\rho_{G, X}(E)\right)=\rho_{G, \mathbb{X}}(\pi(E))$. The proof of part (2) is complete.

Finally, since $\gamma$ is the highest weight of an arbitrary non-trivial $G$-submodule of $H^{0}\left(X, \mathcal{O}_{X}(E)\right)$ and the latter is multiplicity-free since $X$ is spherical, the proof of (3) is also complete.

Notice that property (21) is a rather strong condition on the fan $\mathcal{F}_{G}(X)$. It implies that any maximal cone $c$ of $\mathcal{F}_{G}(X)$ is generated by $\rho_{G, X}(E)$ together with the intersection $c \cap \sigma_{\pi(E)}^{\perp}$.

Example 7.3. Let us illustrate Lemma 7.2 in an example. Consider again $G=\operatorname{SL}(n+1)$ acting on $X=\mathrm{Bl}_{p}\left(\mathbb{P}^{n+1}\right) \times\left(\mathbb{P}^{n}\right)^{*}$ as in Example 2.6. Set $\mathcal{D}=(\partial X)^{\ell}=\left\{D_{1}, D_{2}\right\}$, and recall that $X$ has a third $G$-invariant prime divisor $E$. The prime divisor $\pi(E)=\{([v],[\eta]) \mid \eta(v)=0\}$ of $\mathbb{X}=\mathbb{P}^{n} \times\left(\mathbb{P}^{n}\right)^{*}$ is not stable under the action of $\operatorname{Aut}^{\circ}(\mathbb{X})=\operatorname{PSL}(n+1) \times \operatorname{PSL}(n+1)$. The spherical root $\sigma_{\pi(E)}$ is the unique element $\sigma$ of $\Sigma_{G}(X)$. As in Lemma 7.2, all maximal cones of $\mathcal{F}_{G}(X)$ are generated by $\rho(E)$ together with their intersection with $\sigma^{\perp}$, which is here the linear part of $\mathrm{V}_{G}(X)$.

Definition 7.4. We denote by

$$
\Lambda_{G}(X, \mathcal{E}) \subseteq \Lambda_{G}(X)
$$

the sublattice generated by the elements $\sigma_{\pi(E)}$ for all $E \in \mathcal{E}$.

\section{Corollary 7.5.}

$$
\Lambda_{G}(X)=\rho_{G, X}(\mathcal{E})^{\perp} \oplus \Lambda_{G}(X, \mathcal{E}) .
$$


Proof. From Lemma 7.2 we deduce that for all $E \in \mathcal{E}$ the element $\rho_{G, X}(E)$ is -1 on the spherical root $\sigma_{\pi(E)}$ of $X$, and zero on all other spherical roots of $X$. The corollary follows.

Remark 7.6. In the proof of Lemma 7.2 we used the crucial fact that $X$ and $\mathbb{X}(G / \widehat{H})$ have the same spherical roots. The decomposition of $\Lambda(G / H)$ into the above direct sum would indeed be false in general, if we had used $\mathbb{X}(G / \bar{H})$ instead of $\mathbb{X}(G / \widehat{H})$.

Definition 7.7. Define

$$
\mathbb{E}=\{\pi(E) \mid E \in \mathcal{E}\}
$$

and

$$
\mathbb{D}=\partial \mathbb{X} \backslash \mathbb{E}
$$

Definition 7.8. Let $A^{\prime}=A^{\prime}(X, \mathcal{D})$ be the universal cover of the semisimple group $\operatorname{Aut}^{\circ}(\mathbb{X}, \mathbb{D})$, and $A=A(X, \mathcal{D})=A^{\prime} \times C$. We denote by

$$
\vartheta^{\prime}: G^{\prime} \rightarrow A^{\prime}
$$

the lift of $\theta_{G^{\prime}, \mathbb{X}}: G^{\prime} \rightarrow \operatorname{Aut}^{\circ}(\mathbb{X}, \mathbb{D})$ to $A^{\prime}$, and we set

$$
\vartheta=\vartheta^{\prime} \times \operatorname{id}_{C}: G \rightarrow A
$$

We also choose a Borel subgroup $B_{A}$ of $A$ such that $B_{A} \supseteq \vartheta(B)$.

Now we are ready to state the main result of this section.

Theorem 7.9. Under the assumptions listed at the beginning of this section, and with the above notations:

(1) The action of $A(X, \mathcal{D})$ lifts from $\mathbb{X}$ to $X$, and the image of $A(X, \mathcal{D})$ inside $\operatorname{Aut}^{\circ}(X)$ is equal to $\operatorname{Aut}^{\circ}(X, \mathcal{D})$.

(2) As an $A=A(X, \mathcal{D})$-variety, $X$ is $G$-regular with boundary $\mathcal{D}$.

(3) Via the inclusion $B_{A} \supseteq \vartheta(B)$ the lattice $\Lambda_{A}(X)$ is identified with the lattice $\rho_{G, X}(\mathcal{E})^{\perp} \subseteq$ $\Lambda_{G}(X)$, and $\mathrm{N}_{A}(X)$ with $\Lambda_{G}(X, \mathcal{E})^{\perp} \subseteq \mathrm{N}_{G}(X)$.

(4) The set $\Delta_{A}(X)$ is in natural bijection with $\Delta_{G}(X)$, in such a way that $\rho_{A, X}(D)=$ $\left.\rho_{G, X}(D)\right|_{\rho_{G, X}(\mathcal{E})}$.

(5) The parabolic subgroup $\mathrm{P}_{A}(X)$ is equal to $\mathrm{P}_{A}(\mathbb{X})$, and we have $\Sigma_{A}(X)=\Sigma_{A}(\mathbb{X})$. Both can be computed using Theorem 4.3 and [Pe09, Sections $3.2-3.6]$.

(6) Each cone of $\mathcal{F}_{G}(X)$ intersects $\Lambda_{G}(X, \mathcal{E})^{\perp}$ in a face, and the set of these intersections for all cones of $\mathcal{F}_{G}(X)$ is the fan $\mathcal{F}_{A}(X)$.

The proof occupies the rest of the section. In particular, part (5) follows immediately from part (1). The first statement of part (4) follows from the fact that the $G$-colors and the $A$-colors of $\mathbb{X}$ coincide (see [Br07, Theorem 2.4.2 (2)]), and their inverse images under $\pi$ are the colors of 
$X$. The second statement of part (4) follows from the first, and from part (31). The rest follows from Lemma 7.14, Theorem 7.19, and Corollary 7.20.

Example 7.10. Let us discuss the above theorem in the setting of Example 7.3. Here $\mathbb{D}=\varnothing$, and notice that the action of $\operatorname{Aut}^{\circ}(\mathbb{X}, \mathbb{D})=\operatorname{PSL}(n+1) \times \operatorname{PSL}(n+1)$ does not lift to an action on $X=\mathrm{Bl}_{p}\left(\mathbb{P}^{n+1}\right) \times\left(\mathbb{P}^{n}\right)^{*}$, whereas the action of the universal cover $A^{\prime}=\mathrm{SL}(n+1) \times \mathrm{SL}(n+1)$ does. Moreover $C$ is trivial, so $A=A^{\prime}$.

Under the action of $A$ the variety $X$ is toroidal of rank 1; its lattice is generated by the last fundamental weight of the first factor $\operatorname{SL}(n+1)$ of $A$, and this lattice is identified as in the theorem with the one-dimensional sublattice $\rho_{G, X}(\mathcal{E})^{\perp}=\mathbb{Z} \omega_{n}^{G}$ of $\Lambda_{G}(X)$. The other summand $\Lambda_{G}(X, \mathcal{E})$ of $\Lambda_{G}(X)$ is the lattice $\mathbb{Z} \sigma=\mathbb{Z}\left(\omega_{1}+\omega_{n}\right)$.

As a consequence $\mathrm{N}_{A}(X)$ is identified with the one-dimensional subspace $\sigma^{\perp}$ of $\mathrm{N}_{G}(X)$, the fan $\mathcal{F}_{A}(X)$ is the unique fan with two (opposite) cones of dimension 1, and is obtained from $\mathcal{F}_{G}(X)$ by intersecting all cones with $\sigma^{\perp}$.

In view of proving part (11) of Theorem 7.9, we start finding a candidate for a generic stabilizer of the $A$-action on $X$. Let $\widehat{H}_{A} \subseteq A$ b 3 the stabilizer of the point $e \widehat{H} \in G / \widehat{H} \subseteq \mathbb{X}$. Then we have $\vartheta(\widehat{H})=\widehat{H}_{A} \cap \vartheta(G)$.

We also notice that thanks to our general assumptions any $G$-linearization of an invertible sheaf $\mathbb{X}$ can be uniquely extended to an $A$-linearization, inducing an identification of the two groups $\operatorname{Pic}^{G}(\mathbb{X})$ and $\operatorname{Pic}^{A}(\mathbb{X})$.

Lemma 7.11. (1) The pull-back of characters of $B_{A}$ along $\left.\vartheta\right|_{B}$ induces an injective map $r: \Lambda_{A}(\mathbb{X}) \rightarrow \Lambda_{G}(\mathbb{X})$. It maps $\Sigma_{A}(\mathbb{X})$ to the set of spherical roots $\left\{\sigma_{D} \mid D \in \mathbb{D}\right\}$.

(2) The dual map $r^{*}: \mathrm{N}_{G}(\mathbb{X}) \rightarrow \mathrm{N}_{A}(\mathbb{X})$ satisfies

$$
r^{*}\left(V_{G}(\mathbb{X})\right)=V_{A}(\mathbb{X}) .
$$

(3) We have that $\partial_{A} \mathbb{X}=\mathcal{D}$, and

$$
A / \widehat{H}_{A}=\mathbb{X} \backslash \bigcup_{D \in \mathbb{D}} D .
$$

(4) The pull-back of characters of $\widehat{H}_{A}$ along $\left.\vartheta\right|_{\widehat{H}}$ is a surjective homomorphism $r^{\prime}: \mathcal{X}\left(\widehat{H}_{A}\right) \rightarrow$ $\mathcal{X}(\widehat{H})$ with free kernel of rank $|\mathbb{E}|$.

Proof. The injectivity of the map $r$ is obvious, since it corresponds to taking a $B_{A^{-} \text {-semiinvariant }}$ $f \in \mathbb{C}(\mathbb{X})$ and considering it as a $B$-semiinvariant. The rest of part (1) follows from the results

\footnotetext{
${ }^{3}$ Our notation is consistent thanks to Corollary 7.15.
} 
of [Pe09], and it can also be shown directly using the following fact: the spherical roots of $\mathbb{X}$ are the $T$-weights appearing in the quotient of tangent spaces

$$
\frac{\mathrm{T}_{z} \mathbb{X}}{\mathrm{T}_{z}(G z)}
$$

where $z \in \mathbb{X}$ is the unique fixed point of $B^{-}$. Let us choose a maximal torus $T_{A}$ of $A$ containing $\vartheta(T)$ : if $B_{A}^{-} \subseteq A$ is the Borel subgroup satisfying $B_{A} \cap B_{A}^{-}=T_{A}$ then $B_{A}^{-}$contains $\vartheta\left(B^{-}\right)$. Hence $z$ is also the unique $B_{A}^{-}$-fixed point, therefore the spherical roots of $\mathbb{X}$ as an $A$-variety are the $T_{A}$-weights appearing in the quotient of tangent spaces

$$
\frac{\mathrm{T}_{z} \mathbb{X}}{\mathrm{T}_{z}(A z)}
$$

form the set $\Sigma_{A}(\mathbb{X})$. This implies part (1), and part (2) is an immediate consequence.

The first statement of part (3) stems from the fact that each $E \in \mathbb{E}$ is not stable under the action of $A$, and the second follows from the first because $\mathbb{X}$ is wonderful under the action of A.

For part (4), we notice that $r^{\prime}$ can be identified with the natural map

$$
\frac{\mathcal{X}(C) \times \mathbb{Z}^{\Delta}}{\bar{\rho}_{A, \mathbb{X}}\left(\Lambda_{A}(\mathbb{X})\right)} \rightarrow \frac{\mathcal{X}(C) \times \mathbb{Z}^{\Delta}}{\bar{\rho}_{G, \mathbb{X}}\left(\Lambda_{G}(\mathbb{X})\right)}
$$

(see diagram (3.1)). The kernel of $r^{\prime}$ is then $\Lambda_{G}(\mathbb{X}) / r\left(\Lambda_{A}(\mathbb{X})\right)$ which is free, generated by the spherical roots $\sigma_{E}$ for all $E \in \mathbb{E}$ by part (1).

Let us put together two copies of the diagram (3.1), one for the $G$-and one for the $A$-action, also adding the extensions of $\bar{\rho}_{G}$ and $\bar{\rho}_{A}$ resp. to $\Lambda_{G}(G / H)$ and $\Lambda_{A}\left(A / \widehat{H}_{A}\right)$, as in Section 3 , We obtain a commutative diagram

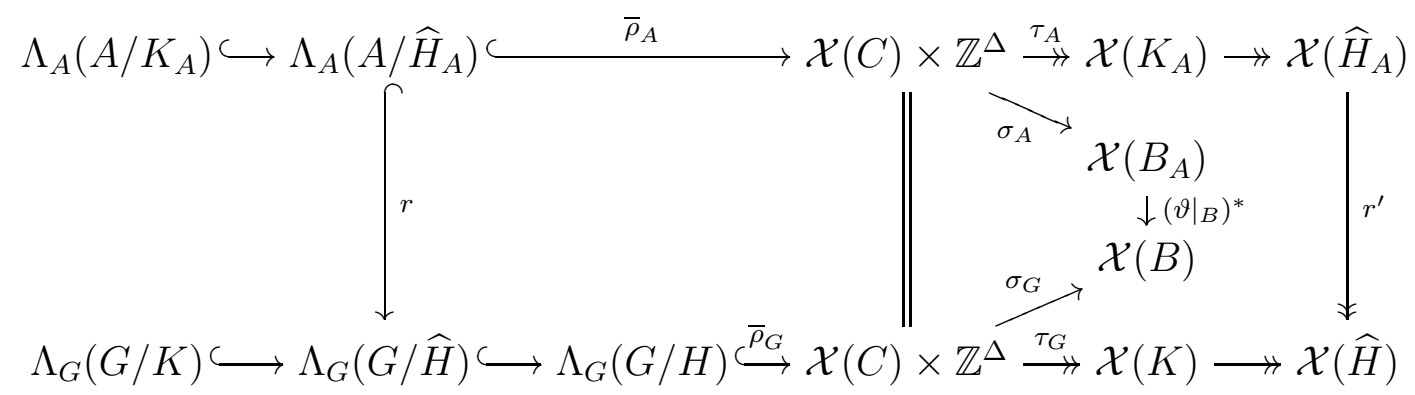

where $K_{A}$ is the spherical closure of $\widehat{H}_{A}$, and $K$ is the spherical closure of $\widehat{H}$ (and of $H$ ). The last arrow of the first row is the restriction map, which can be seen as the quotient

$$
\mathcal{X}\left(K_{A}\right) \rightarrow \mathcal{X}\left(K_{A}\right) / \mathcal{X}\left(K_{A}\right)^{\widehat{H}_{A}} \cong \mathcal{X}\left(\widehat{H}_{A}\right) .
$$

The same remark holds for the last map of the second row and the groups $K, \widehat{H}$.

In order to determine a generic stabilizer in $A$ for $X$, we start defining a lattice $\Lambda \subseteq \Lambda_{G}(G / H)$. A posteriori, it is the lattice of $B$-eigenvalues of $B_{A^{-}}$-eigenvectors $f \in \mathbb{C}(X)^{\left(B_{A}\right)}$. Such a function 
$f$ cannot have zeros nor poles on the divisors in $\mathcal{E}$, since these are not $A$-stable, nor are $A$-colors of $X$. This suggests the following definition of $\Lambda$.

Definition 7.12. Let $\Lambda$ be the lattice

$$
\Lambda=\rho_{G, X}(\mathcal{E})^{\perp} \subseteq \Lambda_{G}(G / H)
$$

Proposition 7.13. The following inclusion holds:

$$
\bar{\rho}_{G}(\Lambda) \supseteq \bar{\rho}_{A}\left(\Lambda_{A}\left(A / K_{A}\right)\right)
$$

The subgroup $H_{A}$ of $K_{A}$ corresponding to the lattice $\bar{\rho}_{G}(\Lambda)$ is a spherical subgroup of $A$. We have $\overline{H_{A}}=K_{A}$ and $\vartheta(H)=H_{A} \cap \vartheta(G)$. This induces a $G$-equivariant identification of $G / H$ with an open subset of $A / H_{A}$.

Proof. Let $\chi \in \Lambda_{A}\left(A / K_{A}\right) \subseteq \Lambda_{A}\left(A / \widehat{H}_{A}\right)$. If $f \in \mathbb{C}\left(A / \widehat{H}_{A}\right)_{\chi}^{\left(B_{A}\right)}$, then consider its pull-back on $X$, denoted by $\tilde{f}$. It is also a $B$-eigenvector with $B$-eigenvalue $\tilde{\chi}=r(\chi)$.

We know that the divisor $\operatorname{div}(\widetilde{f})$ on $X$ is $B_{A}$-stable, so in general it is a linear combination of colors and $A$-stable prime divisors. In any case, its components do not belong to $\mathcal{E}$, because the latter consists of prime divisors moved by $A$. It follows that all discrete valuations in $\mathrm{V}_{G}(G / H)$ coming from these elements of $\mathcal{E}$ must take the value 0 on $\tilde{\chi}$.

Therefore $\tilde{\chi} \in \Lambda$, and the first assertion is proved. In order to verify that $H_{A}$ is spherical we have to show that $\sigma_{A}$ restricted to $\bar{\rho}_{G}(\Lambda)=\tau_{A}^{-1}\left(\mathcal{X}\left(K_{A}\right)^{H_{A}}\right)$ is injective. But we already know that the restriction of $\sigma_{G}$ on $\bar{\rho}_{G}\left(\Lambda_{G}(G / H)\right)$ is injective, and that $\Lambda \subseteq \Lambda_{G}(G / H)$ : this proves the second assertion.

The equality $\overline{H_{A}}=K_{A}$ stems from part (3) of Lemma 3.7, as soon as we check its hypothesis regarding the set $\mathrm{S}_{A}^{\circ}\left(A / K_{A}\right)$, i.e. we have to check that $\sigma_{A}\left(\bar{\rho}_{G}(\Lambda)\right) \cap \mathrm{S}_{A}^{\circ}\left(A / K_{A}\right)=\varnothing$.

Let $\alpha \in \mathrm{S}_{A}^{\circ}\left(A / K_{A}\right)$. We claim that $r(\alpha) \in \mathrm{S}_{G}^{\circ}(G / K)$, i.e. the image $r(2 \alpha)$ is a spherical root $2 \alpha^{\prime}$ of $G / K$ for some simple root $\alpha^{\prime}$ of $G$, such that $\left\langle\alpha^{\prime}, \beta^{\vee}\right\rangle$ is even for all simple root $\beta$ of $G$ such that $2 \beta \in \Sigma_{G}(G / K)$. This stems from an elementary case-by-case check on the classification of [Pe09]: first of all if $2 \alpha$ is a spherical root of $\mathbb{X}$ for the action of $\operatorname{Aut}^{\circ}(\mathbb{X}, \mathbb{D})$ there is an indecomposable factor $\mathbb{X}^{\prime}$ of $\mathbb{X}$ (see [Pe09, Definition 2.2.1]) with $2 \alpha$ among its spherical roots. Then one checks that either the factors of $\theta_{G, \mathbb{X}}(G)$ and of $\operatorname{Aut}^{\circ}(\mathbb{X}, \mathbb{D})$ acting non-trivially on $\mathbb{X}^{\prime}$ are isomorphic, or $\mathbb{X}^{\prime}$ is equal to the case $\boldsymbol{2}_{r k=2}$ of [Pe09].

In the first case we have immediately $r(\alpha) \in \mathrm{S}_{G}^{\circ}(G / K)$. In the second case we have $r(2 \alpha)=$ $2 \alpha^{\prime} \in \Sigma_{G}(\mathbb{X})$ for a simple root $\alpha^{\prime}$ of $G$. Moreover, for all $\beta \in \Sigma_{G}(\mathbb{X})$ the value $\left\langle\alpha^{\prime}, \beta^{\vee}\right\rangle$ is even. This implies again $r(\alpha) \in \mathrm{S}_{G}^{\circ}(G / K)$.

By Lemma 3.7 applied to the inclusion $H \subseteq K$ we have $\sigma_{G}\left(\bar{\rho}_{G}\left(\Lambda_{G}(G / H)\right)\right) \cap \mathrm{S}_{G}^{\circ}(G / K)=\varnothing$. Therefore we have $\sigma_{A}\left(\bar{\rho}_{G}(\Lambda)\right) \cap \mathrm{S}_{A}^{\circ}\left(A / K_{A}\right)=\varnothing$, and hence $\overline{H_{A}}=K_{A}$. 
Next, we claim that $r^{\prime}$ induces an isomorphism between $\mathcal{X}\left(\widehat{H}_{A}\right)^{H_{A}}$ and $\mathcal{X}(\widehat{H})^{H}$. This shows that $\widehat{H}_{A} / H_{A} \cong \widehat{H} / H$, and the rest of the lemma follows. To prove the claim, it is enough to notice that

$$
\begin{aligned}
\mathcal{X}\left(\widehat{H}_{A}\right)^{H_{A}} & \cong \frac{\bar{\rho}_{G}(\Lambda)}{\operatorname{ker} \tau_{A}} \\
& =\frac{\bar{\rho}_{G}(\Lambda)}{\bar{\rho}_{G}\left(r\left(\Lambda_{A}\left(A / \widehat{H}_{A}\right)\right)\right)} \\
& \cong \frac{\bar{\rho}_{G}(\Lambda) \oplus \bar{\rho}_{G}\left(\Lambda_{G}(X, \mathcal{E})\right)}{\bar{\rho}_{G}\left(\Lambda_{G}(G / \widehat{H})\right)} \\
& =\frac{\bar{\rho}_{G}\left(\Lambda \oplus \Lambda_{G}(X, \mathcal{E})\right)}{\bar{\rho}_{G}\left(\Lambda_{G}(G / \widehat{H})\right)} \\
& =\frac{\bar{\rho}_{G}\left(\Lambda_{G}(G / H)\right)}{\bar{\rho}_{G}\left(\Lambda_{G}(G / \widehat{H})\right)} \\
& \cong \mathcal{X}(\widehat{H})^{H}
\end{aligned}
$$

and that the resulting isomorphism $\mathcal{X}\left(\widehat{H}_{A}\right)^{H_{A}} \cong \mathcal{X}(\widehat{H})^{H}$ is indeed induced by $r^{\prime}$.

We build an embedding $X_{A}$ of $A / H_{A}$, and then prove that we actually obtain $X$.

Lemma 7.14. The pull-back of characters of $B_{A}$ to $B$ along $\left.\vartheta\right|_{B}$ induces an injective map $s: \Lambda_{A}\left(A / H_{A}\right) \rightarrow \Lambda_{G}(G / H)$ whose image is $\Lambda$. The dual map $s^{*}: \mathrm{N}_{G}(G / H) \rightarrow \mathrm{N}_{A}\left(A / H_{A}\right)$ satisfies

$$
s^{*}\left(\mathrm{~V}_{G}(G / H)\right)=\mathrm{V}_{A}\left(A / H_{A}\right)
$$

and induces an isomorphism

$$
\left.s^{*}\right|_{\mathrm{V}_{G}^{\ell}(G / H)}: \mathrm{V}_{G}^{\ell}(G / H) \rightarrow \mathrm{V}_{A}^{\ell}\left(A / H_{A}\right) .
$$

Proof. Let $\gamma \in \Lambda_{A}\left(A / H_{A}\right)$ : it is the $B_{A^{-}}$-eigenvalue of a $B_{A^{-}}$-eigenvector $f \in \mathbb{C}\left(A / H_{A}\right)^{\left(B_{A}\right)}$. But $f$ is a $B$-eigenvector too and the character $\chi=s(\gamma)$ is its $B$-eigenvalue. Both the $B$ - and the $B_{A}$-eigenvalue determine $f$ up to a multiplicative constant, hence $s$ is injective.

Consider the commutative diagram

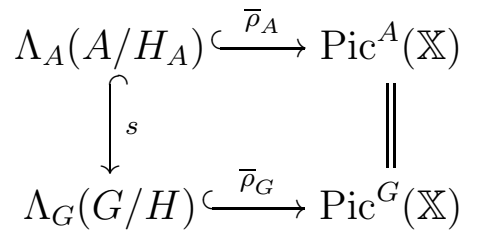

From the definition of $H_{A}$ we have $\bar{\rho}_{A}\left(\Lambda_{A}\left(A / H_{A}\right)\right)=\bar{\rho}_{G}(\Lambda)$, therefore we obtain $s\left(\Lambda_{A}\left(A / H_{A}\right)\right)=$ $\Lambda$. 
Let $v \in \mathrm{V}_{A}\left(A / H_{A}\right)$. It corresponds to an $A$-invariant valuation, which is a fortiori $G$-invariant too: in other words we can compute $v$ also on $\Lambda_{G}(G / H)$ obtaining an element of $\mathrm{V}_{G}(G / H)$. This shows that $s^{*}\left(\mathrm{~V}_{G}(G / H)\right) \supseteq \mathrm{V}_{A}\left(A / H_{A}\right)$.

Then we notice that $s$ extends the map $r$ of Lemma 7.11. This gives the commutative diagram

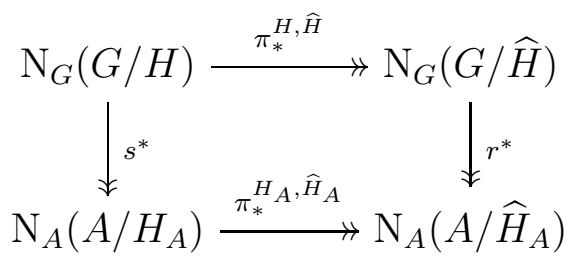

where $V_{A}\left(A / H_{A}\right)\left(\right.$ resp. $\left.V_{G}(G / H)\right)$ is the inverse image of $V_{A}\left(A / \widehat{H}_{A}\right)$ (resp. of $\left.V_{G}(G / \widehat{H})\right)$ thanks to Lemma 3.2 .

This, together with Lemma 7.11, part (2), proves $s^{*}\left(\mathrm{~V}_{G}(G / H)\right)=\mathrm{V}_{A}\left(A / H_{A}\right)$. The image of $\mathrm{V}_{G}^{\ell}(G / H)$ is contained in $\mathrm{V}_{A}^{\ell}\left(A / H_{A}\right)$, and we conclude the proof observing that the dimensions of $\mathrm{V}_{G}^{\ell}(G / H)$ and $\mathrm{V}_{G}^{\ell}\left(A / H_{A}\right)$ are both equal to the dimension of $\widehat{H} / H \cong \widehat{H}_{A} / H_{A}$.

Corollary 7.15. The wonderful closure of $H_{A}$ is $\widehat{H}_{A}$.

Proof. By construction $H_{A} \subseteq \widehat{H}_{A} \subseteq K_{A}=\overline{H_{A}}$. From Lemma 7.14 we deduce that $A / H_{A}$ and $A / \widehat{H}_{A}$ have the same spherical roots: the corollary follows then from Proposition 3.11,

We shall now define the fan of convex cones of $X_{A}$, using that of $X$. First, we collect some consequences on $\mathcal{F}(X)$ of the analysis we have developed so far.

Definition 7.16. Let $\mathcal{F}$ be a fan of convex cones, consider a subset $\mathcal{F}^{\prime} \subset \mathcal{F}$ and let $c \in \mathcal{F} \backslash \mathcal{F}^{\prime}$ be 1-dimensional. Then $\mathcal{F}$ is the join of $\mathcal{F}^{\prime}$ and $c$ if each element of $\mathcal{F} \backslash \mathcal{F}^{\prime}$ is the convex cone generated by $c$ and an element of $\mathcal{F}^{\prime}$.

Corollary 7.17. (1) Let $E \in \mathcal{E}$, and let $\mathcal{F}_{G}^{\sigma_{\pi(E)}}(X)$ be the fan of convex cones obtained intersecting each element of $\mathcal{F}_{G}(X)$ with $\sigma_{\pi(E)}^{\perp}$. Then $\mathcal{F}_{G}(X)$ is the join of $\mathcal{F}_{G}^{\sigma_{\pi(E)}}(X)$ and $c_{X, E}$.

(2) Let $\mathcal{F}_{G}^{\Lambda}(X)$ be the fan of convex cones obtained intersecting each element of $\mathcal{F}_{G}(X)$ with $\Lambda_{G}(X, \mathcal{E})^{\perp}$. Then the restriction of $s^{*}$ to $\operatorname{supp} \mathcal{F}_{G}^{\Lambda}(X)$ is injective, and $s^{*}\left(\operatorname{supp} \mathcal{F}_{G}^{\Lambda}(X)\right)=$ $\mathrm{V}_{A}\left(A / H_{A}\right)$.

(3) The set

$$
\left\{s^{*}(c) \mid c \in \mathcal{F}_{G}^{\Lambda}(X)\right\}
$$

is a fan of polyhedral convex cones in $\mathrm{N}_{A}\left(A / H_{A}\right)$. The associated embedding of $A / H_{A}$ is smooth and complete.

Proof. Part (11) follows from Lemma 7.2, part (2). Part (2) follows from part (11) applied to all $E \in \mathcal{E}$, together with Corollary 7.5 and Lemma 7.14, We turn to part (31). Completeness of 
this embedding is an immediate consequence of part (2). For smoothness, we observe that a maximal cone $c$ of $\mathcal{F}_{G}(X)$ can be written as

$$
c=\left(\left\{-\sigma_{E} \mid E \in \mathcal{E}\right\} \cup \Psi\right)^{\geq 0}
$$

where $\Psi$ is a basis of $\Lambda=\rho_{G, X}(\mathcal{E})^{\perp}$, thanks to the smoothness of $X$ together with part (1) applied to all $E \in \mathcal{E}$ and Corollary 7.5 . Therefore

$$
s^{*}\left(c \cap\left(\Lambda_{G}(X, \mathcal{E})^{\perp}\right)\right)=\left(s^{-1}(\Psi)\right)^{\geq 0} .
$$

The smoothness characterization recalled in Section 2 is verified, since $s^{-1}(\Psi)$ is a basis of $\Lambda_{A}\left(A / H_{A}\right)$, and the proof is complete.

Definition 7.18. We define

$$
\mathcal{F}_{A}=\left\{s^{*}(c) \mid c \in \mathcal{F}_{G}^{\Lambda}(X)\right\},
$$

and we denote by $X_{A}$ the corresponding embedding of $A / H_{A}$.

Theorem 7.19. The inclusion $G / H \subseteq A / H_{A}$ extends to an $A$-equivariant isomorphism between $X$ and $X_{A}$.

Proof. The group $G$ acts on $X_{A}$ via the map $\theta$, and it is enough to show $X_{A}$ is a toroidal embedding of $G / H$ with fan $\mathcal{F}_{G}(X)$. Let us first prove this fact with the assumption that $|\mathcal{E}|=1$, say $\mathcal{E}=\{E\}$.

In addition to the $G$-equivariant map $\pi: X \rightarrow \mathbb{X}$ we also have by construction an $A$ equivariant map $\pi_{A}: X_{A} \rightarrow \mathbb{X}$ extending the projection $\pi^{H_{A}, \widehat{H}_{A}}: A / H_{A} \rightarrow A / \widehat{H}_{A}$. The $A$-colors and the $G$-colors of $\mathbb{X}$ coincide, and this implies the same for $X_{A}$ : indeed any $A$-color (resp. $G$-color) of $X_{A}$ is of the form $\pi_{A}^{-1}(D)$ for an $A$-color (resp. $G$-color) $D$ of $\mathbb{X}$.

If $D \subset \mathbb{X}$ is a color such that $\pi_{A}^{-1}(D)$ contains a $G$-orbit $Y \subset X_{A}$, then $D$ contains the $G$-orbit $\pi_{A}(Y)$ : this is absurd because $\mathbb{X}$ is a toroidal $G$-variety. In other words $X_{A}$ is a toroidal $G$-variety.

Next, we claim that $A / H_{A}$ is a $G$-embedding of $G / H$ whose fan contains $c_{X, E}$ as its unique non-trivial cone. Part (3) of Lemma 7.11 implies that $A / \widehat{H}_{A}$ is an elementary embedding of $G / \widehat{H}$, with orbits $G / \widehat{H}, \pi(E) \cap A / \widehat{H}_{A}$, and fan containing $c_{\mathbb{X}, \pi(E)}$ as its unique non-trivial cone. The open subset $G / H \subset A / H_{A}\left(\subseteq X_{A}\right)$ is equal to $\pi_{A}^{-1}(G / \widehat{H})$, and the $G$-stable closed subset $E^{\prime}=\left(A / H_{A}\right) \backslash(G / H)$ is equal to $\pi_{A}^{-1}(\pi(E)) \cap A / H_{A}$.

Consider the $G$-invariant prime divisors contained in $E^{\prime}$ : they are neither colors nor $A$-stable prime divisors. We claim that there is only one of them, with associated convex cone $c_{X, E}$. Then $E^{\prime}$ itself is a $G$-stable prime divisor, because we already proved that $A / H_{A}$ is a toroidal embedding of $G / H$.

To show the claim, consider $f \in \mathbb{C}(G / H)_{\lambda}^{(B)}$ with $\lambda \in \Lambda$. By Lemma 7.14 we have that $f$ is also a $B_{A}$-eigenvector, therefore its divisor $\operatorname{div}(f)$ on $A / H_{A}$ has components which are either 
colors or $A$-stable prime divisors. It follows that $\rho_{G, A / H_{A}}(F) \in \lambda^{\perp}$ for all $\lambda \in \Lambda$ and all $G$-stable prime divisor $F \subseteq E^{\prime}$. Since $c_{X, E}=\Lambda^{\perp} \cap \mathrm{V}_{G}(G / H)$, we deduce that there is only one such $F$ and it satisfies $\rho_{G, A / H_{A}}(F) \in c_{X, E}$ : the claim above follows.

Now Lemma 7.2. Lemma 7.14 and Corollary 7.17 part (1) hold also if we replace $X$ with $X_{A}$ and $\mathcal{D}$ with the set $\left(\partial_{G} X_{A}\right) \backslash\left\{E^{\prime}\right\}$. From Corollary 7.17 part (11) we deduce that $\mathcal{F}_{G}\left(X_{A}\right)$ is the join of $\mathcal{F}_{G}^{\sigma_{\pi(E)}}\left(X_{A}\right)$ and $c_{X, E}$. From Lemma 7.14 we deduce that every $G$-stable prime divisor $D$ of $X_{A}$ such that $\rho_{G, X_{A}}(D) \in \sigma_{\pi(E)}^{\perp}$ is also $A$-stable, hence each $G$-orbit $Y \subseteq X_{A}$ such that $c_{X_{A}, Y} \subset \sigma_{\pi(E)}^{\perp}$ is also an $A$-orbit.

In other words $\mathcal{F}_{G}^{\sigma_{\pi(E)}}\left(X_{A}\right)$ and $\mathcal{F}_{G}^{\sigma_{\pi(E)}}(X)$ have the same image under $s_{*}$, which implies that they are equal. The theorem in the case $|E|=1$ follows.

If $|\mathcal{E}|>1$, we consider the chain of groups

$$
\theta_{G, X}(G) \subseteq \operatorname{Aut}^{\circ}\left(X, \partial_{G} X \backslash\left\{E_{1}\right\}\right) \subseteq \operatorname{Aut}^{\circ}\left(X, \partial_{G} X \backslash\left\{E_{1}, E_{2}\right\}\right) \subseteq \ldots \subseteq \operatorname{Aut}^{\circ}(X, \mathcal{D}),
$$

where $\mathcal{E}=\left\{E_{1}, E_{2}, \ldots\right\}$, and proceed by induction on $|\mathcal{E}|$. Let $A_{i} \subseteq A_{i+1}$ be two consecutive groups of this chain: we may apply the first part of the proof, together with Corollary 7.21 below (whose proof in the case $|\mathcal{E}|=1$ only depends on the case $|\mathcal{E}|=1$ of this theorem) to the $A_{i}$-variety $X$. We obtain the construction of an $A_{i+1}$-variety $X_{A_{i+1}}$, which is $A_{i}$-equivariantly isomorphic to $X$.

Corollary 7.20. The image of $A$ in $\operatorname{Aut}^{\circ}(X)$ is equal to $\operatorname{Aut}^{\circ}(X, \mathcal{D})$.

Proof. By construction $A$ moves each element of $\mathbb{E}$ on $\mathbb{X}$ and stabilizes all elements of $\mathbb{D}$, hence $\mathbb{D}=\partial_{A} \mathbb{X}$.

Moreover $\widehat{H}_{A}$ is the wonderful closure of $H_{A}$, hence we can apply the exact sequence (4.4) to $X$ as an $A$-variety, mapping onto the wonderful $A$-variety $\mathbb{X}$. Since the image of $A$ contains by construction both the universal cover of $\operatorname{Aut}^{\circ}(\mathbb{X}, \mathbb{D})=\operatorname{Aut}^{\circ}\left(\mathbb{X}, \partial_{A} \mathbb{X}\right)$ and $\left(\widehat{H}_{A} / H_{A}\right)^{\circ} \cong(\widehat{H} / H)^{\circ} \subseteq C$, it follows that the image of $A$ contains $\operatorname{Aut}^{\circ}\left(X, \partial_{A} X\right)=\operatorname{Aut}^{\circ}(X, \mathcal{D})$.

We recall that our assumptions on $\mathcal{D}$ include the fact that all elements of $\mathcal{E}=\partial X \backslash \mathcal{D}$ are not stable under the action of $\operatorname{Aut}^{\circ}(X)$. From the above theorem, together with Corollary 7.20 and the definition of $\mathcal{F}_{A}$, it follows that $\partial_{A} X=\mathcal{D}$, in other words all elements of $\mathcal{E}$ are also not stable under the action of $\operatorname{Aut}^{\circ}(X, \mathcal{D})$. We end this section with the following additional observation that will be useful later.

Corollary 7.21. We have $\left(\partial_{A} X\right)^{\ell}=\left(\partial_{G} X\right)^{\ell}$.

Proof. This is obvious from the definition of $\mathcal{F}_{A}$. 


\section{Abelian case}

In this section we will assume that $G=C$ is an algebraic torus, $X$ as usual a complete $G$-regular variety, and $\mathcal{D} \subseteq \partial_{G} X$ any subset. Hence $X$ is a toric variety under the acton of a quotient of $G$. Since $G$ is its own Borel subgroup, $X$ has no $G$-color. In this setting the study of $\operatorname{Aut}^{\circ}(X)$ is simplified by the fact that, for all $D \in \partial_{G} X$, the $G$-module $H^{0}\left(X, \mathcal{O}_{X}(D)\right)$ splits into the sum of 1-dimensional $G$-submodules.

We report some results on $\operatorname{Aut}^{\circ}(X)$ from [De70], starting with the following proposition on one-dimensional subgroups of $\operatorname{Aut}^{\circ}(X)$. A standard reference on this subject is also Oda88.

Let us recall that any element of $\operatorname{Hom}_{\mathbb{Z}}\left(\Lambda_{G}(X), \mathbb{Z}\right)=\operatorname{Hom}_{\mathbb{Z}}\left(\mathcal{X}\left(\theta_{G, X}(G)\right), \mathbb{Z}\right)$ is canonically associated with a one-parameter subgroup $\mathbb{G}_{m} \rightarrow G$. This induces a tangent vector field on $X$ via the action of $G$, namely the image, via the differential of $\mathbb{G}_{m} \rightarrow \operatorname{Aut}^{\circ}(X)$, of the derivation of $\mathbb{C}\left[\mathbb{G}_{m}\right] \cong \mathbb{C}\left[z, z^{-1}\right]$ defined by $z \mapsto z$.

Proposition 8.1 ([1a88, Section 3.4]). Let $\alpha \in \Lambda_{G}(X)$ be non-zero. The divisor $X(\alpha)$ exists if and only if there exists a one-dimensional unipotent subgroup $U_{\alpha} \subset \operatorname{Aut}^{\circ}(X)$ such that $G$ normalizes $U_{\alpha}$, and the action of $G$ by conjugation on $U_{\alpha} \cong \mathbb{C}$ is linear with weight $\alpha$. If this is the case, then the following holds.

(1) The subgroup $U_{\alpha} \subset \operatorname{Aut}^{\circ}(X)$ is unique, and $X(\alpha)$ is the unique $G$-stable prime divisor of $X$ not stable under the action of $U_{\alpha}$.

(2) Let $c_{\alpha}$ be the one dimensional cone of the fan $\mathcal{F}_{G}(X)$ associated with $X(\alpha)$, let $X_{0}$ be a $G$-stable affine open subset of $X$ and let $c$ be the cone in $\mathcal{F}_{G}(X)$ corresponding to the closed $G$-orbit of $X_{0}$. If $c_{\alpha}$ is an edge of $c$, then $X_{0}$ is $G \ltimes U_{\alpha}$-stable.

(3) Fix an isomorphism $u_{\alpha}: \mathbb{C} \rightarrow U_{\alpha} \subseteq \operatorname{Aut}^{\circ}(X)$ and consider the differential $d u_{\alpha}:$ Lie $\mathbb{C} \rightarrow$ Lie $\operatorname{Aut}^{\circ}(X)$. Then $d u_{\alpha}(d / d \xi)=f_{\alpha} \delta_{\alpha}$, where $d / d \xi$ is the derivation with respect to the coordinate $\xi$ of $\mathbb{C}$, the function $f_{\alpha}$ is in $\mathbb{C}(X)_{\alpha}^{(B)}$, and $\delta_{\alpha}$ is the tangent vector field on $X$ induced by $\rho_{G, X}(X(\alpha))$, seen as an element of $\operatorname{Hom}_{\mathbb{Z}}\left(\mathcal{X}\left(\theta_{G, X}(G)\right), \mathbb{Z}\right)$.

(4) More explicitly, if $f_{\beta} \in \mathbb{C}(X)_{\beta}^{(G)}$ for some $\beta \in \Lambda_{G}(X)$ and $\xi \in U_{\alpha} \cong \mathbb{C}$, then the rational function $z \mapsto f_{\beta}(\xi \cdot z)$ on $X$ is given by the formula

$$
f_{\beta}(\xi \cdot z)=f_{\beta}(z)\left(1+\xi f_{\alpha}(z)\right)^{\left\langle\rho_{G, X}(X(\alpha)), \beta\right\rangle},
$$

(all the G-semiinvariant rational functions are normalized here in such a way that they take value 1 on the same element in the open $G$-orbit of $X)$.

Remark 8.2. If $X(\alpha)$ exists for some $\alpha$ then we have $\left\langle\rho_{G, X}(X(\alpha)), \alpha\right\rangle=-1$ and $\left\langle\rho_{G, X}(D), \alpha\right\rangle \geq$ 0 for all $D \in \partial_{G} X$ different from $X(\alpha)$. However, the difference in signs from our discussion (in particular in Proposition 8.1) and [Oda88, Section 3.4] is only apparent: a character $\lambda \in$ $\mathcal{X}\left(\theta_{G, X}(G)\right)$ is indeed a rational function on $X$ and a $G$-eigenvector, but of $G$-eigenvalue $-\lambda$. 
Notice that the assignment $\alpha \mapsto X(\alpha)$ might be not injective. Also, if both $X(\alpha)$ and $X(-\alpha)$ exist, then $\rho_{G, X}(X(\alpha))$ is not necessarily $-\rho_{G, X}(X(-\alpha))$. However, $X(\alpha)$ and $X(-\alpha)$ are the only $G$-stable prime divisors whose images through $\rho_{G, X}$ are non-zero on $\alpha$.

Definition 8.3. Let $\mathcal{D} \subseteq \partial_{G} X$ any subset, and define $\Phi=\Phi(X, \mathcal{D})$ to be the maximal set of roots of $X$ such that:

(1) if $\alpha \in \Phi(X, \mathcal{D})$ then also $-\alpha \in \Phi(X, \mathcal{D})$;

(2) if $\alpha \in \Phi(X, \mathcal{D})$ then $X(\alpha) \in \mathcal{E}=\partial X \backslash \mathcal{D}$.

The following result is a consequence of Oda88, Demazure's Structure Theorem, Section $3.4]$.

Theorem 8.4. The subgroup of $\operatorname{Aut}^{\circ}(X)$ generated by $\theta_{G, X}(G)$ and $U_{\alpha}$ for all $\alpha \in \Phi(X, \mathcal{D})$ has $\Phi(X, \mathcal{D})$ as root system with respect to its maximal torus $\theta_{G, X}(G)$, and is a Levi subgroup of $\operatorname{Aut}^{\circ}(X, \mathcal{D})$.

Example 8.5. Let $X=\mathbb{P}^{n}$ with $n \geq 2$, under the linear action of the group $G$ of $(n+1) \times(n+1)$ invertible diagonal matrices. Then $\partial_{G} X$ has $n+1$ elements $D_{1}, \ldots, D_{n+1}$ given resp. by the vanishing of the homogeneous coordinates $x_{1}, \ldots, x_{n+1}$. The lattice $\Lambda_{G}(X)$ is the root lattice of the group $\operatorname{GL}(n+1) \supset G$; the simple roots $\alpha_{1}, \ldots, \alpha_{n}$ (with respect to the Borel subgroup of upper triangular matrices and its maximal torus $G$, and in the usual ordering) are the $G$ eigenvalues of the $G$-semiinvariant rational functions $x_{2} / x_{1}, x_{3} / x_{2}, \ldots, x_{n+1} / x_{n}$. In the basis $\alpha_{1}^{*}, \ldots, \alpha_{n}^{*}$ of $\mathrm{N}_{G}(X)$ dual to $\alpha_{1}, \ldots, \alpha_{n}$, the $n+1$ one-dimensional cones in the fan $\mathcal{F}_{G}(X)$ are generated by $\rho\left(D_{1}\right)=-\alpha_{1}^{*}, \rho\left(D_{2}\right)=\alpha_{1}^{*}-\alpha_{2}^{*}, \ldots, \rho\left(D_{n}\right)=\alpha_{n-1}^{*}-\alpha_{n}^{*}, \rho\left(D_{n+1}\right)=\alpha_{n}^{*}$.

The set of all roots of $X$ as a toric variety is the set of roots of $\mathrm{GL}(n+1)$, and the roots $\alpha$ such that $X(\alpha)=D_{n+1}$ are the negative roots with $\alpha_{n}$ in their support. Correspondingly, if $\mathcal{D}=\left\{D_{n+1}\right\}$ then $\operatorname{Aut}^{\circ}(X, \mathcal{D})$ is a maximal parabolic subgroup of $\operatorname{PGL}(n+1)$, its Levi subgroup $A$ containing the image of $G$ is the subgroup whose roots don't have $\alpha_{n}$ in their support.

Notice that $\operatorname{Aut}^{\circ}(X, \mathcal{D})$ acts transitively on $\mathbb{P}^{n} \backslash D_{n+1} \cong \mathbb{C}^{n}$. A one-dimensional "root subgroup" $U_{\alpha}$ contained in the unipotent radical of $\operatorname{Aut}^{\circ}(X, \mathcal{D})$ acts as the translations along one of the coordinates, according to formula (8.1).

Definition 8.6. Define $A=A(X, \mathcal{D})$ the subgroup of $\operatorname{Aut}^{\circ}(X)$ generated by $\theta_{G, X}(G)$ and $U_{\alpha}$ for all $\alpha \in \Phi(X, \mathcal{D})$. Let us also choose a Borel subgroup $B_{A} \subseteq A$ containing $G$ and, consequently, a subdivision of $\Phi$ into positive and negative roots, resp. denoted by $\Phi_{+}=\Phi_{+}(X, \mathcal{D})$ and $\Phi_{-}=\Phi_{-}(X, \mathcal{D})$, and denote by $\Psi=\Psi(X, \mathcal{D})$ the basis of positive roots.

Since $B_{A}$ is generated by $\theta_{G, X}(G)$ together with the subgroups $U_{\alpha}$ for all $\alpha \in \Psi$, we have that any $G$-stable prime divisor which doesn't appear as $X(\alpha)$ for some $\alpha \in \Psi$ is $B_{A}$-stable. In 
other words

$$
\left\{X(\alpha) \mid \alpha \in \Phi_{+}\right\}=\{X(\alpha) \mid \alpha \in \Psi\}
$$

and for the same reason (replacing $\Psi$ with $-\Psi$ )

$$
\left\{X(\alpha) \mid \alpha \in \Phi_{-}\right\}=\{X(\alpha) \mid \alpha \in(-\Psi)\} .
$$

Lemma 8.7. Let $\alpha, \beta \in \Phi$ be different, and suppose that $X(\alpha)=X(\beta)$. Then $\gamma=\alpha-\beta$ and $-\gamma$ are also in $\Phi$, with $X(\gamma)=X(-\beta)$ and $X(-\gamma)=X(-\alpha)$.

Proof. Suppose that $X(-\alpha)=X(-\beta)$. Then $\alpha-\beta$ is zero on $\rho_{G, X}(X( \pm \alpha))$ and on $\rho_{G, X}(X( \pm \beta))$. On the other hand, if a $G$-stable prime divisor $D \subset X$ is not of the form $X( \pm \alpha)$ nor $X( \pm \beta)$, then both $\alpha$ and $\beta$ are zero on $\rho_{G, X}(D)$. It follows that $\operatorname{supp} \mathcal{F}_{G}(X)$ is contained in the hyperplane $(\alpha-\beta)^{\perp}$ of $\mathrm{N}_{G}(X)$, which contradicts the completeness of $X$. Therefore $X(-\alpha) \neq X(-\beta)$, i.e. $X(\alpha), X(-\alpha)$ and $X(-\beta)$ are three different prime divisors. The statement of the lemma is now obvious.

Lemma 8.8. The matrix

$$
\left(\left\langle\rho_{G, X}(X(\alpha)), \alpha^{\prime}\right\rangle\right)_{\alpha, \alpha^{\prime} \in \Psi}
$$

is non-degenerate. In particular, the elements $\rho_{G, X}(X(\alpha))$, for $\alpha$ varying in $\Psi$, are linearly independent.

Proof. Thanks to Lemma 8.7, the elements $\rho_{G, X}(X(\alpha))$ for $\alpha \in \Psi$ are pairwise distinct. If the matrix (8.4) is degenerate, there exists a linear combination

$$
\sum_{\alpha \in \Psi^{\prime}} a_{\alpha} \rho_{G, X}(X(\alpha)) \in \Psi^{\perp}
$$

where $\varnothing \neq \Psi^{\prime} \subseteq \Psi$ and $a_{\alpha} \neq 0$ for all $\alpha \in \Psi^{\prime}$. Applying $\langle\alpha,-\rangle$ for a fixed $\alpha \in \Psi^{\prime}$ to the linear combination (8.5), we see that both $\rho_{G, X}(X(\alpha))$ and $\rho_{G, X}(X(-\alpha))$ must appear in the sum. Indeed, the former appears, and the latter is the only other possible summand that is nonzero on $\alpha$. The elements $\rho_{G, X}(X(-\alpha))$ for $\alpha \in \Psi$ are distinct, thanks to the first part of the proof applied to the set of simple roots $-\Psi$.

Hence each summand in (8.5) can also be rewritten as $a_{\alpha} \rho_{G, X}(X(-\tau(\alpha)))$ where $\tau: \Psi^{\prime} \rightarrow \Psi^{\prime}$ is a bijection. We also know that $\rho_{G, X}(X(\alpha)) \neq \rho_{G, X}(X(-\alpha))$, therefore $\tau$ has no fixed points. Now consider

$$
\gamma=\sum_{\alpha \in \Psi^{\prime}} \alpha
$$

Its value on $\rho_{G, X}(D)$ is zero, if $D \subset X$ is a $G$-stable prime divisor not of the form $X( \pm \alpha)$ for some $\alpha \in \Psi^{\prime}$. On the other hand, for a fixed $\alpha \in \Psi^{\prime}$ we have that $X(\alpha)=X(-\tau(\alpha))$, but 
$X(\alpha) \neq X(\beta)$ for all $\beta \in \Psi$ different from $\alpha$, and $X(\alpha) \neq X(-\beta)$ for any $\beta \in \Psi$ different from $\tau(\alpha)$. Therefore

$$
\begin{aligned}
\left\langle\rho_{G, X}(X(\alpha)), \gamma\right\rangle= & \left\langle\rho_{G, X}(X(\alpha)), \alpha\right\rangle+\left\langle\rho_{G, X}(X(\alpha)), \tau(\alpha)\right\rangle+ \\
& +\left\langle\rho_{G, X}(X(\alpha)), \sum_{\beta \in \Psi^{\prime}, \beta \neq \alpha, \tau(\alpha)} \beta\right\rangle \\
= & -1+1+0=0 .
\end{aligned}
$$

We obtain that $\operatorname{supp} \mathcal{F}_{G}(X)$ is contained in the hyperplane $\gamma^{\perp}$, which is absurd because $X$ is complete.

Proposition 8.9. As an A-variety, $X$ is spherical (not necessarily toroidal). The set of its A-stable prime divisors is

$$
\partial_{A} X=\partial_{G} X \backslash\{X(\alpha) \mid \alpha \in \Phi\},
$$

and these are exactly the G-stable prime divisors $D$ such that $\rho_{G, X}(D) \in \Psi^{\perp}$. Given the identification $\mathcal{X}\left(\theta_{G, X}(G)\right)=\mathcal{X}\left(B_{A}\right)$, we have an inclusion

$$
\iota: \Lambda_{A}(X) \rightarrow \Lambda_{G}(X)
$$

whose image is the sublattice

$$
\left\{\rho_{G, X}(X(\alpha)) \mid \alpha \in \Psi\right\}^{\perp} \subseteq \Lambda(X) .
$$

The restriction map $\iota^{*}: \mathrm{N}_{G}(X) \rightarrow \mathrm{N}_{A}(X)$ induces an isomorphism

$$
\left.\iota^{*}\right|_{\Psi^{\perp}}: \Psi^{\perp} \cong \mathrm{N}_{A}(X) .
$$

For any $B_{A^{-}}$stable prime divisor $D \subset X$ we have $\rho_{A, X}(D)=\iota^{*} \rho_{G, X}(D)$, and the set of $A$-colors of $X$ is the following:

$$
\Delta_{A}(X)=\{X(\alpha) \mid \alpha \in(-\Psi)\} \backslash\{X(\alpha) \mid \alpha \in \Psi\} .
$$

The set of simple roots associated to $\mathrm{P}_{A}(X)$ is

$$
\left\{\alpha \in \Psi \mid X(-\alpha) \notin \Delta_{A}(X)\right\} .
$$

Finally, let $\alpha \in \Psi$ with $X(-\alpha) \in \Delta_{A}(X)$. For all $\beta \in \Phi_{+}$different from $\alpha$, we have $X(-\alpha) \neq$ $X(\beta)$ and $X(\alpha) \neq X(\beta)$. In particular, if in addition $\beta \in \Psi$, we also have $\rho_{G, X}(X(-\alpha)) \in \beta^{\perp}$.

Proof. Since $\theta_{G, X}(G) \subseteq B_{A}$ has already an open orbit on $X$, the first statement is obvious. The statement about the $A$-stable prime divisors is also immediate.

Let us prove that the $A$-colors are the set $\Delta_{A}(X)$ as above defined. A color must be $X(\alpha)$ for some $\alpha \in \Phi$ otherwise it is $A$-stable, and at this point not being of the form $X(\alpha)$ for any $\alpha \in \Phi_{+}$is equivalent to be stable under $B_{A}$. Then, we conclude using (8.2) and (8.3). The statement on the parabolic subgroup $\mathrm{P}_{A}(X)$ is obvious. 
The inclusion $\iota$ is given by the simple observation that a $B_{A}$-eigenvector in $\mathbb{C}(X)$ is a fortiori a $G$-eigenvector, with same eigenvalue; the identity $\rho_{A, X}(D)=\iota^{*} \rho_{G, X}(D)$ for any $B_{A}$-stable prime divisor is also obvious.

Let us prove that the image of $\iota$ is the lattice (8.6). If $\gamma \in \Lambda_{A}(X)$, then a corresponding

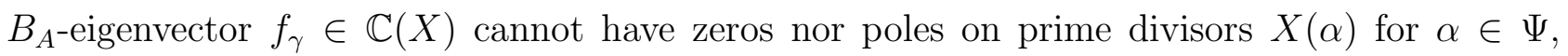

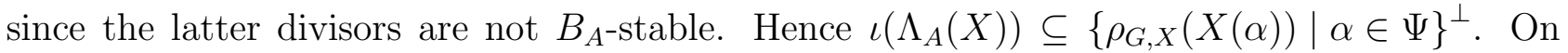
the other hand, if $\chi \in\left\{\rho_{G, X}(X(\alpha)) \mid \alpha \in \Psi\right\}^{\perp}$, then a corresponding $G$-eigenvector $f_{\chi} \in \mathbb{C}(X)$ has zeros and poles only on $A$-stable prime divisors or on colors. It follows that $f_{\chi}$ is also a $B_{A}$-eigenvector, and the other inclusion is proved.

We prove now that $\left.\iota^{*}\right|_{\Psi^{\perp}}$ is an isomorphism between $\Psi^{\perp}$ and $\mathrm{N}_{A}(X)$. From the first part of the proof, this follows if we prove that

$$
\Lambda \otimes_{\mathbb{Z}} \mathbb{Q}=\left(\Psi \otimes_{\mathbb{Z}} \mathbb{Q}\right) \oplus\left(\left\{\rho_{G, X}(X(\alpha)) \mid \alpha \in \Psi\right\}^{\perp} \otimes_{\mathbb{Z}} \mathbb{Q}\right),
$$

and this equality is a consequence of Lemma 8.8 .

Let us check the last statement, so let $\alpha \in \Psi$ be such that $X(-\alpha) \in \Delta_{A}(X)$, and consider $\beta \in \Phi_{+}, \beta \neq \alpha$. We know that $X(-\alpha) \neq X(\beta)$ because of the definition of $\Delta_{A}(X)$ together with (8.2). This also implies that $X(\alpha) \neq X(\beta)$, because otherwise we would have $\beta-\alpha \in \Phi_{+}$ with $X(-\alpha)=X(\beta-\alpha)$, thanks to Lemma 8.7 .

Remark 8.10. The two above results imply in particular that the $A$-colors of $X$, seen as elements of $\mathrm{N}_{A}(X)$, are linearly independent.

Example 8.11. The projective space $X=\mathbb{P}^{n}$, with $\mathcal{D}$ as in Example 8.5 and $n \geq 2$, is not toroidal as an $A$-variety. The group $A \cong \mathrm{GL}(n)$ acts with a fixed point contained in all elements of $\partial_{G} X \backslash \mathcal{D}$, therefore contained in all $A$-colors of $X$.

We can now state the main theorem of this section.

Theorem 8.12. If we identify $\mathrm{N}_{A}(X)$ and $\Psi^{\perp}$ via the map $\left.\iota^{*}\right|_{\Psi^{\perp}}$ of Proposition [8.9, the fan of colored convex cones $\mathcal{F}_{A}(X)$ of $X$ as a spherical $A$-variety is obtained from the fan $\mathcal{F}_{G}(X)$ as follows:

$$
\mathcal{F}_{A}(X)=\left\{\left(c \cap \Psi^{\perp}, d(c)\right) \mid c \in \mathcal{F}_{G}(X)\right\} .
$$

Here $d(c)$ is the set of $A$-colors $D$ of $X$ such that if $\beta \in \Phi_{+}$satisfies $X(-\beta)=D$, then both $\rho_{G, X}(X(\beta))$ and $\rho_{G, X}(X(-\beta))$ lie on 1-dimensional faces of $c$.

Proof. First, we consider $c \in \mathcal{F}_{G}(X)$ and we show that the colored cone $\left(c \cap \Psi^{\perp}, d(c)\right)$ belongs to $\mathcal{F}_{A}(X)$.

The cone $c$ is equal to $c_{X, Y}$ for some $G$-orbit $Y$. We claim that the colored cone associated to the $A$-orbit $A Y$ is given by $\left(c \cap \Psi^{\perp}, d(c)\right)$, with $d(c)$ defined as in the theorem. To show the claim, it is enough to prove that: 
(1) the $A$-stable prime divisors containing $A Y$ are the $G$-stable prime divisors $D$ such that $D \supseteq Y$ and $\rho_{G, X}(D) \in \Psi^{\perp}$

(2) the set of the $A$-colors containing $A Y$ is $d(c)$;

(3) the convex cone $c^{\prime}$ generated by the image of elements of (11) and (2) under the map $\iota^{*} \circ \rho$ is $c \cap \Psi^{\perp}$.

Part (11) is obvious, thanks to the results on $\partial_{A} X$ contained in Proposition 8.9, For part (2), let us first prove that a color $D$ not belonging to $d(c)$ doesn't contain $A Y$. If $D$ doesn't contain $Y$ there is nothing to prove, therefore we may assume that $\rho_{G, X}(D)$ lies on a 1-dimensional face of $c$. Suppose at first that $X(-\beta)=D$ for some $\beta \in \Phi_{+}$, in such a way that $X(\beta)$ doesn't contain $Y$.

Let $X_{c}$ be the affine $G$-stable open subset of $X$ such that its unique closed $G$-orbit has cone c. In other words:

$$
X_{c}=\{x \in X \mid \overline{G x} \supseteq Y\} .
$$

It is isomorphic to an affine space, and thanks to Proposition 8.1 it is stable under the action of $U_{-\beta}$.

More precisely, there exist global coordinates $\left(x_{1}, \ldots, x_{n}\right)$ on $X_{c}$ such that $X(-\beta) \cap X_{c}$ is the hyperplane defined by the equation $x_{1}=0$, and in these coordinates $U_{-\beta}$ acts as follows:

$$
u_{-\beta}(\xi)\left(x_{1}, x_{2}, \ldots, x_{n}\right)=\left(x_{1}+\xi, x_{2}, \ldots, x_{n}\right) .
$$

This formula is checked directly using Proposition 8.1 and the fact that $X(-\beta)$ is the only $G$-stable prime divisor that contains $Y$ and where $\beta$ is non-zero. The hyperplane defined in $X_{c}$ by $x_{1}=0$ contains $Y$, but from (8.7) we deduce that it doesn't contain $U_{\beta} Y$. As a consequence, $A Y$ is not contained in $X(-\beta)$.

Now we show that a color $D$ in $d(c)$ contains $A Y$. At first, consider $\beta \in \Phi_{+}$such that $X(-\beta)=D$. Both $X(-\beta)$ and $X(\beta)$ contain $Y$, and we consider again the affine space $X_{c}$. Applying Proposition 8.1 once again, there exist coordinates $\left(x_{1}, x_{2}, \ldots, x_{n}\right)$ such that $X(-\beta) \cap X_{c}$ is defined by the equation $x_{1}=0$, and $X(\beta) \cap X_{c}$ by the equation $x_{2}=0$, and such that

$$
u_{-\beta}(\xi)\left(x_{1}, x_{2}, \ldots, x_{n}\right)=\left(x_{1}+\xi x_{2}, x_{2}, \ldots, x_{n}\right)
$$

and

$$
u_{\beta}(\xi)\left(x_{1}, x_{2}, \ldots, x_{n}\right)=\left(x_{1}, x_{2}+\xi x_{1}, \ldots, x_{n}\right) .
$$

We obtain that $Y$ is both $U_{-\beta}$-stable and $U_{\beta}$-stable, being contained in the subset of $X_{c}$ defined by $x_{1}=x_{2}=0$. Therefore $X(-\beta)=D$ contains $Y=U_{\beta} U_{-\beta} Y$. 
Now observe that the image of the multiplication map

$$
\prod_{\gamma \in \Phi} U_{\gamma} \times \theta_{G, X}(G) \rightarrow A
$$

(where the product is taken in any fixed order) is dense in $A$. It follows that $D$ contains $A Y$, if we prove that $D$ is $U_{\gamma}$-stable for all $\gamma \in \Phi$ such that $\gamma \neq \pm \beta$ for all $\beta \in \Phi_{+}$satisfying $X(-\beta)=D$. For $\gamma \in \Phi_{-}$there is nothing to prove. But also for $\gamma \in \Phi_{+}$we know that $D \neq X(\gamma)$ : this fact stems from the last statement of Proposition 8.9 together with (8.2). The proof of (2) is complete.

Let us prove (3) . Call $P$ the set of $A$-stable prime divisors containing $A Y$. Then we can describe a minimal set of generators of $c$ (as a convex cone) as the union of the following subsets:

(a) the set $\rho_{G, X}(P)$;

(b) for each color $D \in d(c)$ the set

$$
\left\{\rho_{G, X}(D)\right\} \cup\left\{\rho_{G, X}(X(\beta)) \mid \beta \in \Phi_{+}, X(-\beta)=D\right\}
$$

(c) other generators, different from any of the above.

We show that $c \cap \Psi^{\perp}$ is contained in $c^{\prime}$, and recall that the latter is generated by $\rho_{G, X}(P)$ together with $\iota^{*}\left(\rho_{G, X}(d(c))\right)$. An element $x \in c \cap \Psi^{\perp}$ is a linear combination with non-negative coefficients of the above generators, and we may assume that the elements of (a) do not contribute. This indeed implies the general case, since $\rho_{G, X}(P) \subseteq c^{\prime}$.

Also, we may suppose that any generator $z$ involved in the linear combination giving $x$ satisfies $\iota^{*}(z) \neq 0$. Indeed, otherwise we may suppress it using the fact that $x=\iota^{*}(x)$. Hence, all generators in the linear combination of $x$ are not of the form $\rho_{G, X}(X(\beta))$ for $\beta \in \Psi$.

It remains the generators $\rho_{G, X}(D)$ where $D \in d(c)$, and generators of (c) of the form $\rho_{G, X}(X(-\alpha))$ for some $\alpha \in \Psi$. In the second case $X(-\alpha)$ is a color, because it cannot be equal to $X(\beta)$ for any $\beta \in \Psi$. Being not in $d(c)$, each such $X(-\alpha)$ admits a positive root $\beta$ satisfying $X(-\beta)=X(-\alpha)$ and $D_{\beta}$ not a generator of $c$. This implies that $\beta$ is non-positive on $c$, and the only chance for $x$ to be in $\beta^{\perp}$ is that such a generator $X(-\beta)=X(-\alpha)$ doesn't occur.

As a consequence, $x$ is a linear combination of the elements $\rho_{G, X}(D)$ with $D \in d(c)$, and we conclude that $x \in c^{\prime}$ using again $\iota^{*}(x)=x$.

Finally, let $x \in c^{\prime}$, and let us show that $x \in c \cap \Psi^{\perp}$. As before, we ignore the generators of $c^{\prime}$ lying in $\Psi^{\perp}$, and we assume that $x$ is a linear combination with non-negative coefficients of $\iota^{*}(d(c))$. In other words:

$$
x=\sum_{\alpha \in \Psi, X(-\alpha) \in d(c)} a_{\alpha} \iota^{*}\left(\rho_{G, X}(X(-\alpha))\right)
$$


with $a_{\alpha} \geq 0$. Consider a summand $a_{\alpha} \iota^{*}\left(\rho_{G, X}(X(-\alpha))\right)$. For each positive root $\beta \neq \alpha$ such that $X(-\beta)=X(-\alpha)$, Lemma 8.7 implies that $\gamma=\beta-\alpha$ and $-\gamma$ are also roots in $\Phi$, and that $X(-\alpha)=X(-\beta), X(\alpha)=X(-\gamma), X(\gamma)=X(\beta)$ are three distinct prime divisors. Then, we take the sum

$$
y=\sum_{\alpha \in \Psi, X(-\alpha) \in d(c)} a_{\alpha} y_{\alpha}
$$

where

$$
y_{\alpha}=\rho_{G, X}(X(-\alpha))+\sum_{\substack{\beta \in \Phi_{+}, X(-\beta)=X(-\alpha)}} \rho_{G, X}(X(\beta)) .
$$

We claim that all simple roots in $\Psi$ are zero on this element, hence $\iota^{*}(y)=y$ and we immediately conclude that $y=x$. On the other hand, $y$ is in $c$ thanks to the definition of the set $d(c)$, therefore $x \in c \cap \Psi^{\perp}$.

Let us prove the claim. Let $\gamma \in \Psi$, and pick a $y_{\alpha}$. If $\gamma=\alpha$, then the last assertion of Proposition 8.9 implies that $y_{\alpha}$ is the sum of $\rho_{G, X}(X(-\alpha))$ and $\rho_{G, X}(X(\alpha))$, plus other terms where $\alpha$ is zero. It follows $\left\langle y_{\alpha}, \gamma\right\rangle=0$.

If $\gamma \neq \alpha$, then $\left\langle\rho_{G, X}(X(-\alpha)), \gamma\right\rangle=0$ thanks to Proposition 8.9. Moreover, in this case $\gamma$ does not appear as a $\beta$ in the sum expressing $y_{\alpha}$, because we know that $X(-\alpha) \neq X(-\gamma)$. Also, if $X( \pm \gamma)$ is different from $\rho_{G, X}(X(\beta))$ for all $\beta \in \Phi_{+}$such that $X(-\beta)=X(-\alpha)$, then again $\left\langle y_{\alpha}, \gamma\right\rangle=0$.

Therefore we may suppose that $\gamma$ is different from all the $\beta$ appearing in the expression of $y_{\alpha}$, but some of them, say $\beta_{i, \gamma}$ for $i=1, \ldots, k$, satisfy $X\left(\beta_{i, \gamma}\right)=X\left(\epsilon_{i, \gamma} \gamma\right)$ where $\epsilon_{i, \gamma}=1$ or -1 . In this case Lemma 8.7 implies that $\beta_{i, \gamma}-\epsilon_{i, \gamma} \gamma$ also appears in the sum, with $X\left(\beta_{i, \gamma}-\epsilon_{i, \gamma} \gamma\right)=$ $X\left(-\epsilon_{i, \gamma} \gamma\right)$. We obtain:

$$
\begin{aligned}
y_{\alpha}= & \rho_{G, X}(X(-\alpha))+\sum_{\substack{i=1 \\
\beta \in \Phi_{+}, X(\beta) \neq X( \pm \gamma) \\
X(-\beta)=X(-\alpha)}}^{k}\left(\rho_{G, X}\left(X\left(\beta_{i, \gamma}\right)\right)+\rho_{G, X}\left(X\left(\beta_{i, \gamma}-\epsilon_{i, \gamma} \gamma\right)\right)\right)+ \\
= & \rho_{G, X}(X(-\alpha))+\sum_{i=1}^{k}\left(\rho_{G, X}(X(\gamma))+\rho_{G, X}(X(-\gamma))\right)+ \\
& +\sum_{\substack{\beta \in \Phi_{+}, X(\beta) \neq X( \pm \gamma) \\
X(-\beta)=X(-\alpha)}} \rho_{G, X}(X(\beta)) .
\end{aligned}
$$

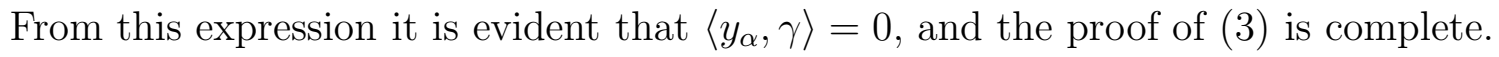

To finish the proof of the theorem, we must check that all colored cones of $\mathcal{F}_{A}(X)$ appear as $\left(c \cap \Psi^{\perp}, d(c)\right)$ for some $c \in \mathcal{F}_{G}(X)$. For this, it is enough to notice that for each $A$-orbit $Z$ there is a $G$-orbit $Y$ such that $A Y=Z$. 
Corollary 8.13. The A-variety is horospherical, i.e. $\Sigma_{A}(X)=\varnothing$.

Proof. There exists a smooth complete toroidal $A$-variety $Y$ equipped with a surjective birational $A$-equivariant morphism $Y \rightarrow X$ (it is enough to choose an $A$-equivariant resolution of singularities of the variety given in [Kn91, Lemma 5.2], where $X^{\prime \prime}$ in the proof of loc.cit. is our $X)$.

Then $\Sigma_{A}(Y)=\Sigma_{A}(X)$, and $Y$ is also a complete $G$-regular embedding. Applying Theorem 8.12 to $Y$, it follows that $\operatorname{supp} \mathcal{F}_{A}(Y)$ is a vector space, and it is equal to $\mathrm{V}_{A}(Y)$ because $Y$ is toroidal and complete. We conclude that $\Sigma_{A}(Y)=\varnothing$.

Remark 8.14. With a slightly more involved proof, one can derive the above corollary directly from Proposition 8.9 and avoid using Theorem 8.12 .

Remark 8.15. Notice that $d(c)=\varnothing$ if and only if $c \cap \Psi^{\perp}$ is a face of $c$. In other words $X$ is toroidal under the action of $A$ if and only if the subspace $\Psi^{\perp}$ intersects each cone of the fan of $X$ in a face of the cone.

Example 8.16. Let us compute the colored fan of $X=\mathbb{P}^{2}$, as in Example 8.5 with $n=2$. We consider again the set $\mathcal{D}=\left\{D_{3}\right\}$, so $\mathcal{E}=\left\{D_{1}, D_{2}\right\}$, where $D_{i}$ is given by the vanishing of the homogeneous coordinate $x_{i}$. We have $D_{1}=X\left(\alpha_{1}\right)=X\left(\alpha_{1}+\alpha_{2}\right), D_{2}=X\left(\alpha_{2}\right)=X\left(-\alpha_{1}\right)$ and $D_{3}=X\left(-\alpha_{1}-\alpha_{2}\right)=X\left(-\alpha_{2}\right)$. Then $A \subset \operatorname{Aut}^{\circ}\left(\mathbb{P}^{2}, \mathcal{D}\right)$ is isomorphic to $\mathrm{GL}(2)$ with roots $\alpha_{1}$ and $-\alpha_{1}$; choosing the Borel subgroup of $A$ stabilizing the point $[1,0,0]$ corresponds to choosing $\Psi=\left\{\alpha_{1}\right\}$. The lattice $\Lambda_{A}\left(\mathbb{P}^{2}\right)$ is $\rho_{G, \mathbb{P}^{2}}\left(D_{1}\right)^{\perp}=\mathbb{Z} \alpha_{2}$, and $\mathbb{P}^{2}$ has only one $A$-color, namely $D_{2}$. The maximal colored cones of the original fan $\mathcal{F}_{G}\left(\mathbb{P}^{2}\right)$ are the cones $c_{i, j}$ generated by $\rho_{G, \mathbb{P}^{2}}\left(D_{i}\right)$ and $\rho_{G, \mathbb{P}^{2}}\left(D_{j}\right)$ for all $i<j \in\{1,2,3\}$.

The intersection of $c_{1,3}$ and $c_{2,3}$ with $\Psi^{\perp}$ is the half line $\mathbb{Q}_{\geq 0}$ if we identify $\mathrm{N}_{A}\left(\mathbb{P}^{2}\right)$ with

using the basis dual to $\alpha_{2} \in \Lambda_{A}\left(\mathbb{P}^{2}\right)$. This intersection is a face of both cones, so this yields the maximal colored cone $\left(\mathbb{Q}_{\geq 0}, \varnothing\right)$ of the fan $\mathcal{F}_{A}\left(\mathbb{P}^{2}\right)$. The intersection of $c_{1,2}$ with $\Psi^{\perp}$ corresponds to $\mathbb{Q}_{\leq 0}$, and it is not a face of $C_{1,2}$; correspondingly, the unique $A$-color $D_{2}$ satisfies the condition required to be in $d\left(c_{1,2}\right)$. This yields the other maximal colored cone of $\mathcal{F}_{A}\left(\mathbb{P}^{2}\right)$, namely $\left(\mathbb{Q}_{\leq 0},\left\{D_{2}\right\}\right)$.

\section{Semisimple CASE}

In this section we assume that $G$ is a semisimple group, i.e. $C=\{e\}$. In this setting the functionals associated to the colors of $X$ generate $\mathrm{N}_{G}(X)$ as a vector space. Indeed, if $\lambda \in \Lambda_{G}(X)$ is in $\rho_{G, X}\left(\Delta_{G}(X)\right)^{\perp}$, then a rational function $f \in \mathbb{C}(G / H)_{\lambda}^{(B)}$ is regular on $G / H$ and nowhere zero. It can be then lifted to a nowhere-vanishing function $F \in \mathbb{C}[G]$, which is then constant since $G$ has no non-trivial character (see [KKV89, Proposition 1.2]). We conclude that $\lambda=0$, and the claim follows. 
This essentially implies the following main result of this section.

Theorem 9.1. If $G$ is semisimple and $\mathcal{D}$ is any subset of $\partial_{G} X$, then $\operatorname{Aut}^{\circ}\left(X, \mathcal{D} \cup\left(\partial_{G} X\right)^{\ell}\right)$ is a Levi subgroup of $\operatorname{Aut}^{\circ}(X, \mathcal{D})$.

The proof is at the end of this section. The theorem implies that if $G$ is semisimple then Section 7 is enough to describe a Levi subgroup of $\operatorname{Aut}^{\circ}(X, \mathcal{D})$ and its action on $X$, without any restriction on $\mathcal{D}$.

Recall from Section 6 the restriction map

$$
\kappa_{x^{\prime}}:\left(\operatorname{ker} \psi_{*}\right)^{\circ} \rightarrow \operatorname{Aut}^{\circ}\left(X_{x^{\prime}}\right)
$$

where $x^{\prime}$ lies on the open $G$-orbit of $X^{\prime}$, and $X_{x^{\prime}}=\psi^{-1}\left(x^{\prime}\right)$.

Lemma 9.2. For all $x^{\prime}$ in the open B-orbit of $X^{\prime}$, the image of $\kappa_{x^{\prime}}$ in $\operatorname{Aut}^{\circ}\left(X_{x^{\prime}}\right)$ is connected and solvable.

Proof. The image $\kappa_{x^{\prime}}$ is connected since $\left(\operatorname{ker} \psi_{*}\right)^{\circ}$ is connected; we prove it's solvable. To simplify notations we assume that $x^{\prime}=x_{0}^{\prime}$. Let $\mathcal{E}^{\prime} \subseteq \partial_{S} X_{x_{0}^{\prime}}$ be the following subset:

$$
\mathcal{E}^{\prime}=\left\{E \cap X_{x_{0}^{\prime}} \mid E \in \mathcal{E}\right\}
$$

and define $\mathcal{D}^{\prime}=\partial_{S} X_{x_{0}^{\prime}} \backslash \mathcal{E}^{\prime}$. Let us also denote by $K_{x_{0}^{\prime}}$ the image of $\kappa_{x_{0}^{\prime}}$ : it is obviously a subgroup of $\operatorname{Aut}^{\circ}\left(X_{x_{0}^{\prime}}, \mathcal{D}^{\prime}\right)$. On the other hand $K_{x_{0}^{\prime}}$ contains the maximal torus $S$ of $\operatorname{Aut}^{\circ}\left(X_{x_{0}^{\prime}}\right)$, hence we only have to compute the root subgroups it contains. Thanks to Lemma 6.5 and Corollary [5.5, they are the root spaces $U_{\alpha} \subset \operatorname{Aut}^{\circ}\left(X_{x_{0}^{\prime}}\right)$ for $\alpha$ varying in the set

$$
R=\left\{\left.\gamma\right|_{S} \mid 0 \neq \gamma \in \Lambda_{G}(G / H), X(\gamma) \text { exists and } X(\gamma) \in \mathcal{E}^{\ell}\right\}
$$

From Lemma 6.6, we obtain that $R$ doesn't contain the opposite of any of its elements, therefore $K_{x_{0}^{\prime}}$ is solvable.

Example 9.3. Let $X$ and $G$ be as in Example 7.3. Recall that the $G$-stable prime divisors $D_{1}$, $D_{2}$, and $E$ have valuations resp. $(-1,1),(1,-1)$ and $(-1,0)$ with respect to the basis given by the two colors, and that the first two are those in $(\partial X)^{\ell}$. As in the proof of Lemma 9.2, there exists no $\gamma \in \Lambda_{G}(X)$ such that $X(\gamma)=D_{1}$ and $X(-\gamma)=D_{2}$.

Proof of Theorem 9.1. First, observe that $\left(\operatorname{ker} \psi_{*}\right)^{\circ}$ is solvable. This stems from Lemma 9.2, and the obvious observation that

$$
\bigcap_{x^{\prime} \text { in the open } B \text {-orbit of } X^{\prime}} \operatorname{ker}\left(\kappa_{x^{\prime}}\right)=\left\{\operatorname{id}_{X}\right\} .
$$

Consider now the variety $X$ under the action of $A=\operatorname{Aut}^{\circ}\left(X, \mathcal{D} \cup\left(\partial_{G} X\right)^{\ell}\right)$. Thanks to Theorem 7.9 , the group $A$ is semisimple (because here $G$ is semisimple) and under its action $X$ is 
a $G$-regular embedding with boundary $\mathcal{D} \cup\left(\partial_{G} X\right)^{\ell}$. Corollary 7.21 implies $\left(\partial_{A} X\right)^{n \ell}=\mathcal{D}^{n \ell} \subseteq \mathcal{D}$, and we deduce that $\operatorname{Aut}^{\circ}(X, \mathcal{D}) \subseteq \operatorname{Aut}^{\circ}\left(X,\left(\partial_{A} X\right)^{n \ell}\right)$.

Then we may apply Proposition 5.4 with $G$ replaced by the universal cover of $A$ : the theorem follows.

Remark 9.4. Let $X$ and $G$ be as in Example 7.3. Then $\operatorname{Aut}^{\circ}(X)$ is not reductive. Indeed, it must fix the point $p \in \mathbb{P}^{n+1}$, and it is elementary to conclude that $\operatorname{Aut}^{\circ}(X)$ is the corresponding maximal proper parabolic subgroup of $\operatorname{PGL}(n+2) \times \operatorname{PGL}(n+1)$. The unipotent radical $\operatorname{Aut}^{\circ}(X)^{u}$ can be studied restricting its elements to the generic fiber $X_{x_{0}^{\prime}}$; however, the example shows that for any given fiber the restriction may be non-injective, therefore a global analysis of these restrictions is needed. This goes beyond the scope of the present work.

\section{Prime Divisors on the linear part of the valuation cone}

In this section $G=G^{\prime} \times C$ is neither abelian nor semisimple. Thanks to Section 8, we may assume that $G^{\prime}$ acts non-trivially on $X$. The variety $X^{\prime}$ is then not a single point.

Our goal is to study $\operatorname{Aut}^{\circ}(X, \mathcal{D})$ for a general $\mathcal{D}$. Denote as usual $\mathcal{E}=\partial_{G} X \backslash \mathcal{D}$.

We begin applying the results of Section 7 to the group $\widetilde{G}=\operatorname{Aut}^{\circ}\left(X, \mathcal{D} \cup\left(\partial_{G} X\right)^{\ell}\right)$. From Theorem 7.9 and Corollary 7.21 it follows that $X$ is a regular $\widetilde{G}$-variety with boundary $\mathcal{D} \cup$ $\left(\partial_{G} X\right)^{\ell}$, and the valuations of the elements of $\left(\partial_{G} X\right)^{\ell}$ lie on the linear part of the valuation cone both with respect to the $G$-action and to the $\widetilde{G}$-action on $X$.

Therefore we may replace $G$ with the group $\widetilde{G}$, and proceed to our analysis on $\operatorname{Aut}^{\circ}(X, \mathcal{D})$ where now $X$ is a complete $G$-regular embedding satisfying $\mathcal{D} \supseteq\left(\partial_{G} X\right)^{n \ell}$.

Recall that $S$ acts on $X$ naturally by $G$-equivariant automorphisms preserving the fibers of $\psi$, so we can consider $S$ as a subgroup of $\operatorname{Aut}^{\circ}\left(X, \partial_{G} X\right) \cap\left(\operatorname{ker} \psi_{*}\right)^{\circ}$.

Proposition 10.1. Let $x^{\prime}$ in the open $G$-orbit of $X^{\prime}$, and $L=L(X, \mathcal{D})$ be a Levi subgroup of $\left(\operatorname{Aut}^{\circ}(X, \mathcal{D}) \cap \operatorname{ker}\left(\psi_{*}\right)\right)^{\circ}$ containing $S$. Then $L_{x^{\prime}}=\kappa_{x^{\prime}}(L)$ is isomorphic to $L$, and the group

$$
\left(\theta_{G, X}(G), \theta_{G, X}(G)\right) \times L(X, \mathcal{D})
$$

is locally isomorphic to a Levi subgroup of $\operatorname{Aut}^{\circ}(X, \mathcal{D})$.

Proof. Thanks to formula (9.1) and the fact that $x^{\prime}$ is generic in $X^{\prime}$, we know that the map $L \rightarrow$ $L_{x^{\prime}}$ has unipotent kernel, therefore is an isomorphism. The rest follows from Proposition 5.4.

Recall that we have chosen a base point $x_{0} \in X$ in the open $B$-orbit of $X$, and we denote by $x_{0}^{\prime}$ its image in $X^{\prime}$.

Definition 10.2. We define the following group:

$$
A=A(X, \mathcal{D})=\left(\theta_{G, X}(G), \theta_{G, X}(G)\right) \times L(X, \mathcal{D}),
$$


where $L(X, \mathcal{D})$ is defined as in Proposition 10.1 choosing $x^{\prime}=x_{0}^{\prime}$.

We describe now the reductive group $L_{x_{0}^{\prime}}$ in terms of the root subspaces it contains with respect to its maximal torus $S$.

Definition 10.3. We define

$$
R=R(X, \mathcal{D})=\left\{\left.\gamma\right|_{S} \mid 0 \neq \gamma \in \Lambda_{G}(G / H), X(\gamma) \text { exists and } X(\gamma) \in \mathcal{E}\right\},
$$

and we denote by $\Phi=\Phi(X, \mathcal{D})$ the maximal subset of $R$ such that $-\alpha \in R$ for every $\alpha \in R$.

Proposition 10.4. The set $\Phi(X, \mathcal{D})$ is a subset of $\Phi\left(X_{x_{0}^{\prime}}, \mathcal{D}^{\prime}\right)$, where $\mathcal{D}^{\prime}=\left\{D \cap X_{x_{0}^{\prime}} \mid D \in\right.$ $\left.\mathcal{D}^{\ell}\right\}$. Moreover, $L_{x_{0}^{\prime}} \subseteq \operatorname{Aut}^{\circ}\left(X_{x_{0}^{\prime}}\right)$ is generated by $S$ together with all subgroups $U_{\alpha}$ such that $\alpha \in \Phi(X, \mathcal{D})$.

Proof. For the first assertion, it is enough for any $\alpha=\left.\gamma\right|_{S} \in \Phi(X, \mathcal{D})$ to restrict the function $f \in H^{0}\left(X, \mathcal{O}_{X}(X(\gamma))\right)_{\gamma}^{(B)}$ to $X_{x_{0}^{\prime}}$. Since $S$ is a maximal torus of $\operatorname{Aut}^{\circ}\left(X_{x_{0}^{\prime}}\right)$, the second assertion follows from Lemma 6.5 and Corollary 5.5 .

This provides a complete description of the group $A$. It remains now to describe the fan associated to $X$ as an $A$-variety.

Let $0 \neq \gamma \in \Lambda(G / H)$ be such that $\left.\gamma\right|_{S}=\alpha \in \Phi$, and choose an element $f_{\gamma} \in H^{0}\left(X, \mathcal{O}_{X}(X(\gamma))\right)_{\gamma}^{(B)}$ such that $f_{\gamma}\left(x_{0}\right)=1$. Then $\rho_{G, X}(X(\gamma)) \in \mathrm{V}_{G}^{\ell}(G / H)$ can be considered as an element of $\operatorname{Hom}_{\mathbb{Z}}(\mathcal{X}(S), \mathbb{Z})$, and therefore it is canonically associated with a one-parameter subgroup $\mu_{\gamma}: \mathbb{G}_{m} \rightarrow S$. The torus $S$ acts on $X$ through an identification with a subtorus of $T_{G, X}$, thanks to Lemma 6.4, As in Section 8, we consider the induced tangent vector field on $X$ and we denote it by $\delta_{\gamma} \in H^{0}\left(X, \mathcal{T}_{X}\right)$.

Lemma 10.5. The product $\xi_{\gamma}=f_{\gamma} \delta_{\gamma}$ is a well-defined tangent vector field of $X$, and it is sent to $H^{0}\left(X, \mathcal{O}_{X}(X(\gamma))\right)$ via the surjective map of 4.1). Its restriction to $X_{x_{0}^{\prime}}$ is a tangent vector field and is a generator of the Lie algebra of $U_{\alpha} \subset \operatorname{Aut}^{\circ}\left(X_{x_{0}^{\prime}}\right)$. Moreover, the action of the one-parameter subgroup of $\operatorname{Aut}^{\circ}\left(\mathcal{Z}_{G, X}\right)$ induced by $\xi_{\gamma}$ can expressed using formula (8.1) of Proposition 8.1 .

Proof. Formula (8.1) of Proposition 8.1 holds for $\xi_{\gamma}$ being simply an elementary reformulation of the definition $\xi_{\gamma}=f_{\gamma} \delta_{\gamma}$.

The rational function $f_{\gamma}$ has its only pole in $X(\gamma)$, which means that we only have to check the first assertion on points of $X(\gamma)$. On $\mathcal{Z}_{G, X} \cap X(\gamma)$ it can be checked using the fact that $\mathcal{Z}_{G, X}$ is a toric $T_{G, X}$-variety, and expressing $\xi_{\gamma}$ in local coordinates. This also implies that $\xi_{\gamma}$ is a well-defined vector field on $E \cap X_{0}$, thanks to the $P_{G, X^{u}}^{u}$-invariance of both $f_{\gamma}$ and $\delta_{\gamma}$. Then the locus where $\xi_{\gamma}$ might not be a well-defined vector field has codimension at least 2 , which implies the first statement. 
Since $S$ acts on $X$ stabilizing both $\mathcal{Z}_{G, X}$ and $X_{x_{0}^{\prime}}$, we deduce that $\xi_{\gamma}$ can be restricted to a vector field on both these varieties. The rest follows by expressing $\xi_{\gamma}$ on $\mathcal{Z}_{G, X}$ explicitly in local coordinates.

Definition 10.6. We choose a Borel subgroup $B_{A}$ of $A$ such that $\theta_{A, X}\left(B_{A}\right) \cap \theta_{G, X}(G)=\theta_{G, X}(B)$ and such that $B_{A} \cap L$ is a Borel subgroup of $L$. Let us also denote by $\Psi=\Psi(X, \mathcal{D}) \subset \Phi(X, \mathcal{D})$ the set of simple roots and by $\Phi_{+}=\Phi_{+}(X, \mathcal{D}) \subset \Phi(X, \mathcal{D})$ the set of positive roots associated to the Borel subgroup $B_{L_{x_{0}^{\prime}}}=\kappa_{x_{0}^{\prime}}\left(B_{A} \cap L\right)$ of $L_{x_{0}^{\prime}}$, with respect to the maximal torus $S$. Finally, let

$$
r: \Lambda_{G}(X) \rightarrow \mathcal{X}(S)=\Lambda_{S}\left(X_{x_{0}^{\prime}}\right)
$$

be the restriction of characters of $\Lambda_{G}(X)$ to $S$ (see Section 5 ).

We may apply Proposition 8.9 and Theorem 8.12 to the toric $S$-variety $X_{x_{0}^{\prime}}$ and the sets of roots $\Phi$ and $\Psi$. We obtain a description of $X_{x_{0}^{\prime}}$ as an $L_{x_{0}^{\prime}}$-variety, and in particular the lattice

$$
\Lambda_{L_{x_{0}^{\prime}}}\left(X_{x_{0}^{\prime}}\right) \subseteq \Lambda_{S}\left(X_{x_{0}^{\prime}}\right),
$$

together with the projection

$$
\mathrm{N}_{S}\left(X_{x_{0}^{\prime}}\right) \rightarrow \mathrm{N}_{L_{x_{0}^{\prime}}}\left(X_{x_{0}^{\prime}}\right) .
$$

Proposition 10.7. The restriction of weights from $\theta_{A, X}\left(B_{A}\right)$ to $\theta_{G, X}(B)$ induces an isomorphism

$$
\Lambda_{A}(X) \cong r^{-1}\left(\Lambda_{L_{x_{0}^{\prime}}}\left(X_{x_{0}^{\prime}}\right)\right) \subseteq \Lambda_{G}(X) .
$$

We denote the corresponding surjective map by

$$
s: \mathrm{N}_{G}(X) \rightarrow \mathrm{N}_{A}(X) .
$$

The set of colors of $X$ as a spherical A-variety is the following disjoint union:

$$
\Delta_{A}(X)=\Delta_{G}(X) \cup\left\{E \in \mathcal{E} \mid E \cap X_{x_{0}^{\prime}} \in \Delta_{L_{x_{0}^{\prime}}}\left(X_{x_{0}^{\prime}}\right)\right\},
$$

and for each $E \in \Delta_{A}(X)$, we have

$$
\rho_{A, X}(E)=s\left(\rho_{G, X}(E)\right) .
$$

The simple roots associated to the parabolic subgroup $\mathrm{P}_{A}(X)$ are the union of the set of those associated to $\mathrm{P}_{G}(X)$ with the set $\left\{\alpha \in \Psi \mid X_{x_{0}^{\prime}}(-\alpha) \notin \Delta_{L_{x_{0}^{\prime}}}\left(X_{x_{0}^{\prime}}\right)\right\}$.

Proof. A $B_{A^{-}}$-eigenvector in $\mathbb{C}(X)$ is a fortiori a $B$-eigenvector, thanks to the choice of $B_{A}$. This induces an inclusion $\Lambda_{A}(X) \subseteq \Lambda(X)$.

Moreover, a $B$-eigenvector $f \in \mathbb{C}(X)$ is also a $B_{A}$-eigenvector if and only if its restriction $\left.f\right|_{X_{x_{0}^{\prime}}}$ is a $B_{L_{x_{0}^{\prime}}}$-eigenvector, thanks to the structure of $A$ as described in Proposition 10.1. This proves the first assertion. 
Secondly, a color of $X$ as an $A$-variety maps either dominantly onto $X^{\prime}$, or not. In the first

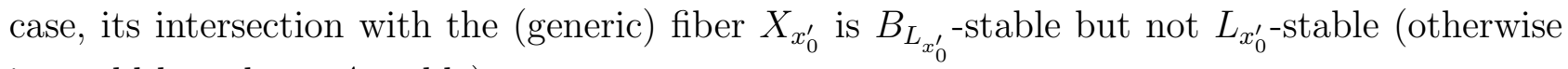
it would have been $A$-stable).

In the second case, it maps onto a $G$-color of $X^{\prime}$, i.e. it is a color of $X$ with respect to the $G$ action. The second assertion follows, and implies the last assertion on $\mathrm{P}_{A}(X)$.

Example 10.8. Let $G=\left(\mathbb{G}_{m}\right)^{2} \times \mathrm{SL}(2)$ act on $Y=\mathbb{P}^{1} \times \mathbb{P}^{1} \times \mathbb{P}^{1}$ as follows: $\left(\mathbb{G}_{m}\right)^{2}$ acts only on the first factor $\mathbb{P}^{1}=\mathbb{P}\left(\mathbb{C}^{2}\right)$ linearly on the two homogeneous coordinates, SL $(2)$ acts on the second and third factor diagonally. Then consider $X$ to be the projective completion of the line bundle $\mathcal{O}_{\mathbb{P}^{1}}(1) \otimes \mathcal{O}_{\mathbb{P}^{1}}(-1) \otimes \mathcal{O}_{\mathbb{P}^{1}}$ on $Y$. Then $X$ is a spherical $G$-variety, with open orbit $G / H$ where

$$
H=\left\{\left(a, b,\left(\begin{array}{cc}
a & 0 \\
0 & a^{-1}
\end{array}\right)\right) \mid a, b \in \mathbb{C} \backslash\{0\}\right\} .
$$

The variety $X$ is toroidal under the action of $G$, the associated wonderful variety is $\mathbb{P}^{1} \times \mathbb{P}^{1}$ and the map $X \rightarrow \mathbb{X}$ extending $G / H \rightarrow G / \widehat{H}$ is the projection of $X$ onto $Y$ followed by the projection $Y \rightarrow \mathbb{X}$ on the second and third coordinate. There are two $G$-colors in $X$, namely the inverse images of the two $G$-colors of $\mathbb{X}$.

There are five $G$-stable prime divisors in $X$, two of them given by the conditions that the projection on the first coordinate of $Y$ is a $\left(\mathbb{G}_{m}\right)^{2}$-stable point (i.e. 0 or $\infty$ in the usual coordinates). These two $G$-stable prime divisors $E_{1}$ and $E_{2}$ are mapped surjectively onto $\mathbb{X}$, therefore setting $\mathcal{D}=\partial_{G} X \backslash \mathcal{E}$ where $\mathcal{E}=\left\{D_{1}, D_{2}\right\}$ we have $\mathcal{D} \supset\left(\partial_{G} X\right)^{n \ell}$.

A Levi subgroup $A$ of $\operatorname{Aut}^{\circ}(X, \mathcal{D})$ (actually, the whole group in this case) is the image of $\mathrm{GL}(2) \times \mathrm{SL}(2)$, where we let $\mathrm{GL}(2)$ act on $X$ by lifting its linear action on the first factor $\mathbb{P}^{1}$ of $Y$. The $A$-colors of $X$ are the $G$-colors together with one additional prime divisor, namely $D_{1}$ or $D_{2}$ (according to the choice of a Borel subgroup of GL(2)).

Let $c$ be a cone of the fan $\mathcal{F}(X)$. Then $c$ is generated as a convex cone by a set of 1-dimensional faces $F(c)$. We denote by $c^{\ell}$ the intersection $c \cap \mathrm{V}_{G}^{\ell}(X)$, by $F^{\ell}(c)$ the 1-dimensional faces of $F(c)$ generating $c^{\ell}$, and $F^{n \ell}(c)=F(c) \backslash F^{\ell}(c)$.

Since $c^{\ell}$ is a cone of the toric $S$-variety $X_{x_{0}^{\prime}}$, it corresponds to an $S$-orbit $Y$ on $X_{x_{0}^{\prime}}$. As

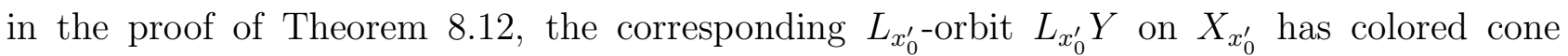
$\left(c^{\ell} \cap \Psi^{\perp}, d\left(c^{\ell}\right)\right)$, where the orthogonal $\Psi^{\perp}$ is taken inside $\mathrm{V}_{G}^{\ell}(G / H)$, and $d\left(c^{\ell}\right)$ is a set of $L_{x_{0}^{\prime}}$ colors of $X_{x_{0}^{\prime}}$.

Definition 10.9. For any $c \in \mathcal{F}(X)$ we define a colored cone $\left(c_{A}(c), d_{A}(c)\right)$, where $c_{A}(c) \subset$ $\mathrm{N}_{A}(X)$ and $d_{A}(c) \subseteq \Delta_{A}(X)$, as follows. The cone $c_{A}(c)$ is the convex cone in $\mathrm{N}_{A}(X)$ generated by $s\left(F^{n \ell}(c)\right)$ and $s\left(c^{\ell} \cap \Psi^{\perp}\right)$. The set $d_{A}(c)$ is the set of colors $E \in \Delta_{A}(X)$ such that $E \notin \Delta_{G}(X)$ and $E \cap X_{x_{0}^{\prime}} \in d\left(c^{\ell}\right)$. 
Theorem 10.10. The colored fan $\mathcal{F}_{A}(X)$ of $X$ as a spherical A-variety is

$$
\mathcal{F}_{A}(X)=\left\{\left(c_{A}(c), d_{A}(c)\right) \mid c \in \mathcal{F}_{G}(X)\right\} .
$$

Proof. Let $Y$ be a $G$-orbit of $X$, with associated cone $c=c_{X, Y}$. We claim that the colored cone associated to the $A$-orbit $A Y$ is $\left(c_{A}(c), d_{A}(c)\right)$ : arguing as in the proof of Theorem 8.12, this is enough to show the theorem.

To prove the claim, first we show that the set $d^{\prime}$ of $A$-colors containing $A Y$ is equal to $d_{A}(c)$. Since $X$ is toroidal, no $G$-color contains $Y$, nor $A Y$. Therefore any $A$-color $E$ in $d^{\prime}$ is indeed a $G$-stable prime divisor whose functional lies in $\mathrm{V}_{G}^{\ell}(X)$. It intersects $X_{x_{0}^{\prime}}$ in an $L_{x_{0}^{\prime}}$-color of $X_{x_{0}^{\prime}}$, by Proposition 10.7, and we only have to show that $E \cap X_{x_{0}^{\prime}}$ is in $d\left(c^{\ell}\right)$.

We check this fact using the definition of $d\left(c^{\ell}\right)$. Take a positive $\operatorname{root} \beta \in \Phi_{+}$of $X_{x_{0}^{\prime}}$, the prime divisors $X_{x_{0}^{\prime}}(\beta), X_{x_{0}^{\prime}}(-\beta)$ of $X_{x_{0}^{\prime}}$, and suppose that $X_{x_{0}^{\prime}}(-\beta)=E \cap X_{x_{0}^{\prime}}$, so $\rho_{S, X_{x_{0}^{\prime}}}\left(X_{x_{0}^{\prime}}(-\beta)\right)$ lies on a 1-codimensional face of $c^{\ell}$. We have to show that $\rho_{S, X_{x_{0}^{\prime}}}\left(X_{x_{0}^{\prime}}(\beta)\right)$ also lies on a 1codimensional face of $c^{\ell}$, in other words that $X_{x_{0}^{\prime}}(\beta)$ contains the $S$-orbit of $X_{x_{0}^{\prime}}$ associated $c^{\ell}$.

Now $E=E_{1}$ and some other element $E_{2} \in \mathcal{E}$ satisfy $E_{1} \cap X_{x_{0}^{\prime}}=X_{x_{0}^{\prime}}(-\beta), E_{2} \cap X_{x_{0}^{\prime}}=X_{x_{0}^{\prime}}(\beta)$, and $-\beta$ and $\beta$ are the restrictions to $S$ of resp. $\gamma_{1}, \gamma_{2} \in \Lambda_{G}(X)$, such that $X\left(\gamma_{i}\right)=E_{i}$ for $i=1,2$. Suppose that $E_{2}$ doesn't contain $Y$. Then we consider $\mathcal{Z}_{G, X}$ : intersecting it with $E_{1}, E_{2}$ and $Y$ two $T_{G, X}$-stable prime divisors and a $T_{G, X}$-orbit, such that $E_{1} \cap \mathcal{Z}_{G, X} \supseteq Y \cap \mathcal{Z}_{G, X}$ and $E_{2} \cap \mathcal{Z}_{G, X} \nsupseteq Y \cap \mathcal{Z}_{G, X}$.

At this point we follow the same approach of the proof of Theorem 8.12, statement (21), applied to the toric variety $\mathcal{Z}_{G, X}$ and the automorphisms induced by the tangent vector field $\xi_{\gamma_{1}}$ (as defined in Lemma 10.5). This yields the formula (8.7) for $\xi_{\gamma_{1}}$, which shows that $E_{1} \cap \mathcal{Z}_{G, X}$ doesn't contain $A Y \cap \mathcal{Z}_{G, X}$ : a contradiction. As a consequence $E_{2} \supseteq Y$, so $X_{x_{0}^{\prime}}(\beta)$ contains the $S$-orbit of $X_{x_{0}^{\prime}}$ associated $c^{\ell}$. This concludes the proof of the inclusion $d^{\prime} \subseteq d_{A}(c)$.

Let now $D \in d_{A}(c)$. Then, by Theorem 8.12, the intersection $D \cap X_{x_{0}^{\prime}}$ contains the $L_{x_{0}^{\prime}}$ orbit of $X_{x_{0}^{\prime}}$ corresponding to $\left(c^{\ell} \cap \Psi^{\perp}, d\left(c^{\ell}\right)\right)$. Let $y$ be a point on this orbit: then $D$ contains $\overline{A y}$.

On the other hand, from the proof of Theorem 8.12, we see that $\overline{L_{x_{0}^{\prime}} y}$ contains the $S$-orbit of $X_{x_{0}^{\prime}}$ corresponding to $c^{\ell} \subset \mathrm{N}_{S}\left(X_{x_{0}^{\prime}}\right)$. It follows that $\overline{A y}$ contains the $G$-orbit of $X$ associated to $c^{\ell} \subset \mathrm{N}_{G}(X)$, and thus also the $G$-orbit $Y$ associated to $c \subset \mathrm{N}_{G}(X)$. Being $A$-stable, $\overline{A y}$ must then contain $A Y$ too, and since $D$ is closed, we obtain $D \supseteq A Y$. I.e., $D$ is in $d^{\prime}$.

We now prove that the convex cone $c^{\prime}$ associated to $A Y$ is $c_{A}(c)$. First observe that $Y$ and $A Y$ are contained in the same elements of $\left(\partial_{G} X\right)^{n \ell}$, since $L$ stabilizes all fibers of $\psi$. Therefore $c^{\prime}$ is generated by $s\left(F^{n \ell}(c)\right)$ and its intersection with $s\left(\mathrm{~V}_{G}^{\ell}(X)\right)$. It remains to prove that $c^{\prime} \cap s\left(\mathrm{~V}_{G}^{\ell}(X)\right)=s\left(c^{\ell} \cap \Psi^{\perp}\right)$.

The cone $c^{\prime} \cap s\left(\mathrm{~V}_{G}^{\ell}(X)\right)$ is generated by $\rho_{A, X}(E)$ where $E \in\left(\partial_{G} X\right)^{\ell}$ is: 
(1) an $A$-color of $X$ containing $A Y$, i.e. $E \in d_{A}(c)$, or

(2) an $A$-stable prime divisor containing $A Y$.

On the other hand the generators of $s\left(c^{\ell} \cap \Psi^{\perp}\right)$ are the elements $\rho_{A, X}(E)$ where $E \in\left(\partial_{G} X\right)^{\ell}$ is:

(1') an $A$-color such that $E \cap X_{x_{0}^{\prime}}$ is a color containing the $L_{x_{0}^{\prime}}$-orbit $Z$ of $X_{x_{0}^{\prime}}$ associated to $\left(c^{\ell} \cap \Psi^{\perp}, d\left(c^{\ell}\right)\right)$, or

$\left(2^{\prime}\right)$ an $A$-stable prime divisor such that $E \cap X_{x_{0}^{\prime}}$ is a $L_{x_{0}^{\prime}}$-stable prime divisor containing $Z$. Thanks to the first part of the proof, the prime divisors $E$ of type (1) and of type (1') are the same.

If $E$ is of type (2') then it contains $A Z$, whose closure in turn contains $A Y$. Therefore $E$ is of type (2). Let now $E$ be of type (2). Then $E \cap \mathcal{Z}_{G, X}$ is an $L$-stable (and $T_{G, X}$-stable) prime divisor of $\mathcal{Z}_{G, X}$ containing $Y \cap \mathcal{Z}_{G, X}$, which is the $T_{G, X}$-orbit of $\mathcal{Z}_{G, X}$ associated with $c$, and

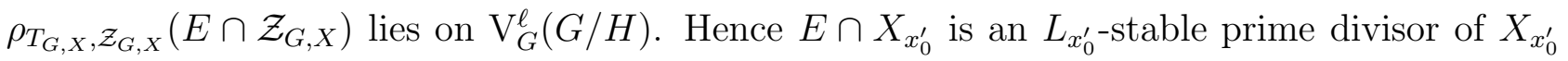
containing the $S$-orbit of $X_{x_{0}^{\prime}}$ associated with $c^{\ell}$. Thanks to the proof of Theorem 8.12 , we deduce that $E \cap X_{x_{0}^{\prime}}$ contains $Z$, i.e. $E$ is of type (2').

Corollary 10.11. $\Sigma_{A}(X)=\Sigma_{G}(X)$.

Proof. The proof is similar to the proof of Corollary 8.13 .

Remark 10.12. Although a Levi subgroup $A$ of $\operatorname{Aut}^{\circ}(X, \mathcal{D})$ splits (up to central isogeny) into a product $\left(\theta_{G, X}(G), \theta_{G, X}(G)\right) \times L(X, \mathcal{D})$, Example 10.8 shows that the open $A$-orbit of $X$ in general is not the product of a $(G, G)$-homogeneous space and an $L(X, \mathcal{D})$-homogeneous space. On the other hand, from Corollary 10.11 it follows that the factor $L(X, \mathcal{D})$ acts trivially on $\mathbb{X}$, whose open $A$-orbit is then always a homogeneous space under the action of $(G, G)$.

\section{REFERENCES}

[BB96] F. Bien, M. Brion: Automorphisms and local rigidity of regular varieties, Compositio Math. 104 (1996), no. $1,1-26$.

[BDP90] E. Bifet, C. De Concini, C. Procesi: Cohomology of regular embeddings, Adv. in Math. 82 (1990), $1-34$.

[BL11] P. Bravi, D. Luna: An introduction to wonderful varieties with many examples of type $\mathrm{F}_{4}$, J. Algebra 329 (2011), 4-51.

[Br89] M. Brion: Groupe de Picard et nombres caractéristiques des variétés sphériques. Duke Math. J. 58 (1989), no. 2, 397-424.

[Br90] M. Brion: Vers une généralisation des espaces symétriques, J. Algebra 134 (1990), no. 1, 115-143.

[Br97] M. Brion: Variétés sphériques,

http://www-fourier.ujf-grenoble.fr/〜mbrion/spheriques.ps

[Br07] M. Brion: The total coordinate ring of a wonderful variety, J. Algebra 313 (2007), no. 1, 61-99.

[BP87] Brion, F. Pauer. Valuations des espaces homogènes sphériques. Commentarii Mathematici Helvetici 62 (1987), no. 2, 265-285. 
[De70] M. Demazure, Sous-groupes algébriques de rang maximum du groupe de Cremona, Ann. Sci. École Norm. Sup. 3, (1970), no. 4, 507-588.

[De77] M. Demazure, Automorphismes et déformations des variétés de Borel, Invent. Math. 39 (1977), no. 2, $179-186$.

[Gi89] V. Ginzburg: Admissible modules on a symmetric space, Astérisque 173-174 (1989), 199-255.

[Kn91] F. Knop: The Luna-Vust theory of spherical embeddings, Proceedings of the Hyderabad Conference on Algebraic Groups (Hyderabad, 1989), 225-249, Manoj Prakashan, Madras, 1991.

[Kn94] F. Knop: The assymptotic behaviour of invariant collective motion, Invent. Math. 114, $1994,309-328$.

[Kn96] F. Knop: Automorphisms, root systems, and compactifications of homogeneous varieties, J. Amer. Math. Soc. 9 (1996), no. 1, 153-174.

[KKLV89] F. Knop, H. Kraft, D. Luna, T. Vust: Local properties of algebraic group actions, in Algebraische Transformationsgruppen und Invariantentheorie (H. Kraft, P. Slodowy, T. Springer eds.) DMV-Seminar 13, Birkhäuser Verlag (Basel-Boston) (1989) 63-76.

[KKV89] F. Knop, H. Kraft, T. Vust: The Picard group of a G-variety, in Algebraische Transformationsgruppen und Invariantentheorie (H. Kraft, P. Slodowy, T. Springer eds.) DMV-Seminar 13, Birkhäuser Verlag (Basel-Boston) (1989) 77-87.

[Lu01] D. Luna: Variétés sphériques de type A, Inst. Hautes Études Sci. Publ. Math. 94 (2001), $161-226$.

[Oda88] T. Oda: Convex Bodies and Algebraic Geometry: An Introduction to Toric Varieties, Springer-Verlag, New York-Berlin-Heidelberg, 1988.

[Pe09] G. Pezzini: Automorphisms of wonderful varieties, Transform. Groups 14 (2009), no. 3, 677-694.

[Wa96] B. Wasserman: Wonderful varieties of rank two, Transform. Groups 1 (1996), no. 4, 375-403.

Departement Mathematik, Friedrich-Alexander Universität Erlangen-Nürnberg, CauerSTRASSE 11, 91058 ERlANGEN, DEUtSCHLAND

E-mail address: pezzini@math.fau.de 